1

\title{
Empirical Characterization of the SMOS Brightness Temperature Bias and Uncertainty for Improving Sea Surface Salinity Retrieval
}

\author{
Estrella Olmedo ${ }^{\circledR}$, Verónica González-Gambau ${ }^{\circledR}$, Antonio Turiel ${ }^{\circledR}$, Member, IEEE,

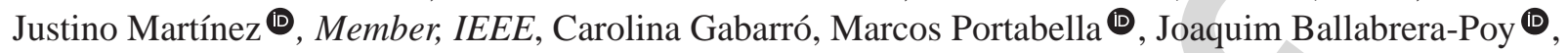 \\ Manuel Arias, Roberto Sabia, Member, IEEE, and Roger Oliva, Member, IEEE
}

\begin{abstract}
After more than eight years of the European Space Agency (ESA) Soil Moisture and Ocean Salinity (SMOS) acquisitions, an exhaustive, empirical characterization of the biases and uncertainties affecting SMOS brightness temperatures over the ocean is possible. We show that both parameters strongly depend not only on the position in the field of view, but also on the geographical location of the acquisition. Metrics based on the differences between expected and theoretical values of the bias and the uncertainty are developed and used for quantitatively assessing the locations where SMOS errors are currently not accurately characterized. This characterization can be used for the definition of a new empirical SMOS sea surface salinity (SSS) bias correction, a better cost function retrieval, and more accurate filtering criteria, which are expected to lead to a better SMOS SSS Level 2 product. We present a new L2 SMOS SSS product based on the described investigation. The performance of this preliminary product is similar to that of the version v662 of the official L2 SMOS SSS product at medium and low latitudes. However, it provides a better coverage at high latitudes and coastal regions affected by radio frequency interference (RFI), which correspond to those regions where the SMOS errors are currently poorly estimated.
\end{abstract}

Index Terms-Brightness temperature (TB) biases, brightness temperature uncertainty, coastal areas, radio frequency interference (RFI), sea surface salinity (SSS), Soil Moisture and Ocean Salinity (SMOS).

Manuscript received September 14, 2018; revised February 14, 2019; accepted March 2, 2019. This work was supported in part by the Ministry of Economy and Competitiveness, Spain, through the National R+D Plan under PROMISES Project ESP2015-67549-C3-R, L-Band Project ESP2017-89463-C3-1-R, and previous grants, and in part by the European Space Agency by means of the contracts SMOS ESL L2OS, CCI+ Salinity, Arctic+ Salinity, and Baltic+ Salinity Dynamics. (Corresponding author: Estrella Olmedo.)

E. Olmedo, V. González-Gambau, A. Turiel, J. Martínez, C. Gabarró, M. Portabella, and J. Ballabrera-Poy are with the Department of Physical Oceanography, Institute of Marine Sciences, CSIC and with the Barcelona Expert Center, Passeig Maritim de la Barceloneta, Barcelona 08003, Spain (e-mail: olmedo@icm.csic.es; vgonzalez@icm.csic.es; turiel@icm.csic.es; justino@ icm.csic.es; cgabarro@icm.csic.es; portabella@icm.csic.es; joaquim@icm. csic.es).

M. Arias is with the Argans, Plymouth PL6 8BX, U.K. (e-mail: MArias@ argans.co.uk)

R. Sabia is with the Telespazio Vega, U.K. Ltd., European Space Agency, ESA-ESRIN, Frascati 00044, Italy (e-mail: roberto.sabia@esa.int).

R. Oliva is with the European Space Agency, ESA-ESAC, Madrid 28692, Spain (e-mail: roger.oliva.balague@esa.int).

Color versions of one or more of the figures in this paper are available online at http://ieeexplore.ieee.org.

Digital Object Identifier 10.1109/JSTARS.2019.2904947

\section{INTRODUCTION}

$\mathbf{M}$ ORE than eight years after its launch, the European Space Agency (ESA) Soil Moisture and Ocean Salinity (SMOS) mission continues to provide global observations of two key variables for the increased knowledge of the earth's water cycle: Soil Moisture and Ocean Surface salinity [1]-[3].

The SMOS instrument, the Microwave Imaging Radiometer using Aperture Synthesis (MIRAS), is an interferometer radiometer that measures snapshots of brightness temperature (TB) [4]. Each acquired TB value corresponds to a given geolocation with a different observation angle (incidence angle). As the satellite moves, each point on the ground is measured multiple times in successive snapshots. This gives SMOS a multiangular capability: for each given geographical location and satellite overpass many different TBs, each one corresponding to a different incidence angle, are acquired. The set of all TB observations associated with a given geographical location and overpass time is called a dwell line. The nominal SMOS retrieval scheme combines the information from all TBs in the same dwell line and their corresponding radiometric accuracies, both of which depend on the incidence angle [5], [6]. This retrieval scheme, also called the Bayesian scheme, is statistically efficient as it increases the accuracy of the retrieved sea surface salinity (SSS) for unbiased TBs. However, it is very sensitive to systematic errors in TB. Unfortunately, SMOS is affected by several systematic errors in TB that substantially degrade the quality of the SSS retrieval [7].

In order to mitigate systematic antenna biases, the application of the so-called ocean target transformation (OTT) was proposed in [8]. The OTT correction intends to address global offsets, which can arise from a calibration or image reconstruction errors or an overall constant offset in the radiative transfer model. This correction consists in removing the mean of the difference between the measured and the modeled TB in a geophysically stable open-ocean region $\Omega$, which corresponds to the area 121 $\pm 16 \mathrm{~W} @ 5 \mathrm{~S}$ and $111 \pm 16 \mathrm{~W} @ 45 \mathrm{~S}$, for ascending overpasses and 112 $\pm 16 \mathrm{~W} @ 5 \mathrm{~S}$ and 123 $\pm 16 \mathrm{~W} @ 45 \mathrm{~S}$ for the descending ones. This correction assumes that the spatial biases are constant over the ocean and they are dependent on antenna patterns and other image reconstruction errors. Although this correction resulted in a great improvement that allowed SSS to 
be retrieved from the beginning of the mission in the vast majority of the global ocean, it was soon found that temporal and other geographically dependent biases were present. In particular, strong biases close to the coasts (the so-called land-sea contamination, LSC) degrade SMOS SSS, making all values closer than $800 \mathrm{~km}$ to the coast largely unreliable. The latest improvements in the official ESA SMOS L2OS processor (v662) include an empirical correction to mitigate the LSC, which consists in removing the mean difference between the measurement and a TB model (before the SSS retrieval), and not always allow the current state of the ocean to be correctly described.

Some other empirical spatial corrections have been proposed to correct the LSC and remaining biases. Studies such as [9] and [10], and more recently [11] have proposed empirical LSC corrections based on the characterization of the retrieved SSS as a function of the geographical location, the direction of the satellite overpass, and the position of the dwell line. The main issue with this kind of corrections is that they are only applied after the standard Bayesian retrieval is performed. Therefore, the presence of some degraded TB in a given dwell line may lead the Bayesian retrieval scheme to diverge, even if that dwell line contains some valid TBs.

A different approach was proposed in [12], where SSS is characterized not only as a function of the geographical location, the direction of the satellite overpass, and the dwell line, but also of the incidence angle of the acquisition. The authors proposed a SSS retrieval scheme in which every TB leads to a single value of SSS (non-Bayesian retrieval). This allows a characterization of the systematic biases on SSS as a function of all the acquisitions and geographical conditions. In this case, the characterization of biases is done on SSS and not on TBs. One of the main advantages is that this non-Bayesian retrieval forces just the degraded SSS (or TBs) to be discarded from the dwell line of a given geographical location. Another advantage of the non-Bayesian scheme is that we can easily translate the errors in SSS to errors in TB. The underlaying hypothesis is that, since the geophysical model function (GMF) monotonously decreases with increasing SSS, once the incidence angle and the other geophysical parameters are fixed, the characterization of the errors can be done in the same way at both SSS and TB levels. That is, since a single value of SSS is retrieved from a single TB, the errors can be translated from SSS space to TB space. Characterizing the errors in the TB space enables the linkage with OTT, LSC, and other effects known to be present in SMOS TB images. Moreover, a better characterization of SMOS errors could enable us to improve the quality of the retrievals in terms of a better coverage and lower error.

The aim of this paper is to use the non-Bayesian scheme to estimate the biases and the uncertainties associated with TBs. Our goal is to describe the source of the errors in SMOS TBs and make use of this knowledge to improve the retrieval of SSS. We use the retrieval approach proposed in [12] and further develop the following aspects. In [12], the characterization of the biases is done at a salinity level; while here, we characterize the SMOS errors (biases and uncertainties) at a TB level. This in turn enables the comparison between our empirically derived biases and the OTT used in the L2OS processor and between the empirical estimation of uncertainties with the radiometric accuracy. We show that regions where the third- and fourth-order moments of long-term salinity retrieval series degrade are associated to regions that are affected by non-permanent radio frequency interferences and other effects such as the eclipse. Finally, in [12], a level 3 product (nine-day map) is proposed. In this study, we define a Level 2 (daily) product. The filtering criteria have been modified to better capture the salinity dynamics. A SMOS SSS daily product can have an added value for applications such as the computation of sea surface currents from salinity fields.

The structure of the paper is as follows. In Section II, we use the methodology of [12] for the empirical bias characterization of the TBs and we estimate how far this empirical bias correction is from the standard OTT correction. In Section III, we estimate an empirical TB uncertainty and we prove that there is an excess of TB uncertainty that cannot be explained either by radiometric or geophysical effects, and that it depends on the geographical location of the retrieval. SMOS TB are also affected by other non-systematic sources of error such as the latitudinalseasonal biases and radio frequency interference (RFI) [7]. The main characteristic of these kinds of error is that they vary with time, and so they are difficult to characterize and to correct. In Section IV, we show that these non-systematic error sources have an impact on the third- and fourth-order moments of the SSS distributions. Based on these assessments, in Section V, a methodology to compute an alternative Level 2 SMOS SSS product is proposed. The aim is to obtain a better product that has higher accuracy and broader coverage, especially in those regions where we demonstrate that the current approach can be improved. Section VI presents a quality assessment of the new product and a comparison with the current version of the official L2OS product (v662). Finally, in Section VII, we discuss the main results presented in this paper.

\section{EMPIRICAL CHARACTERIZATION OF THE SYSTEMATIC BRIGHTNESS TEMPERATURE BIASES}

Following the approach proposed in [12], six years (20112016) of SMOS L1B (v620) data have been used to generate six years of raw SSS data. The L1B product is distributed by the ESA and is freely available at: https://earth.esa.int/web/guest/-/howto-obtain-data-7329. The sun glint [13] and surface roughness [14] contributions are corrected using auxiliary information provided by ECMWF [15], similarly to what is done in the official ESA SMOS L2 SSS products. The galactic reflection [16] is corrected using a compound map built from radio-astronomy observatories. For every First Stokes TB measurement, a single value of SSS has been retrieved following the non-Bayesian scheme. These SSS retrievals have been classified as a function of the satellite overpass direction $(d$, that can be either ascending or descending), the latitude $(\varphi)$, the longitude $(\lambda)$, the across-track distance (i.e., the position in the dwell line, $x$ ), and the incidence angle $(\theta)$ of the acquisition measurement. The fundamental hypothesis of this approach is that, given a fixed value for the tuple $\gamma=(\varphi, \lambda, d, x, \theta)$, the set of SSS retrieved under those particular conditions are characterized by the same errors. Therefore, we have an ensemble of sets $\left\{\mathrm{SSS}_{\gamma}\right\}$ as 

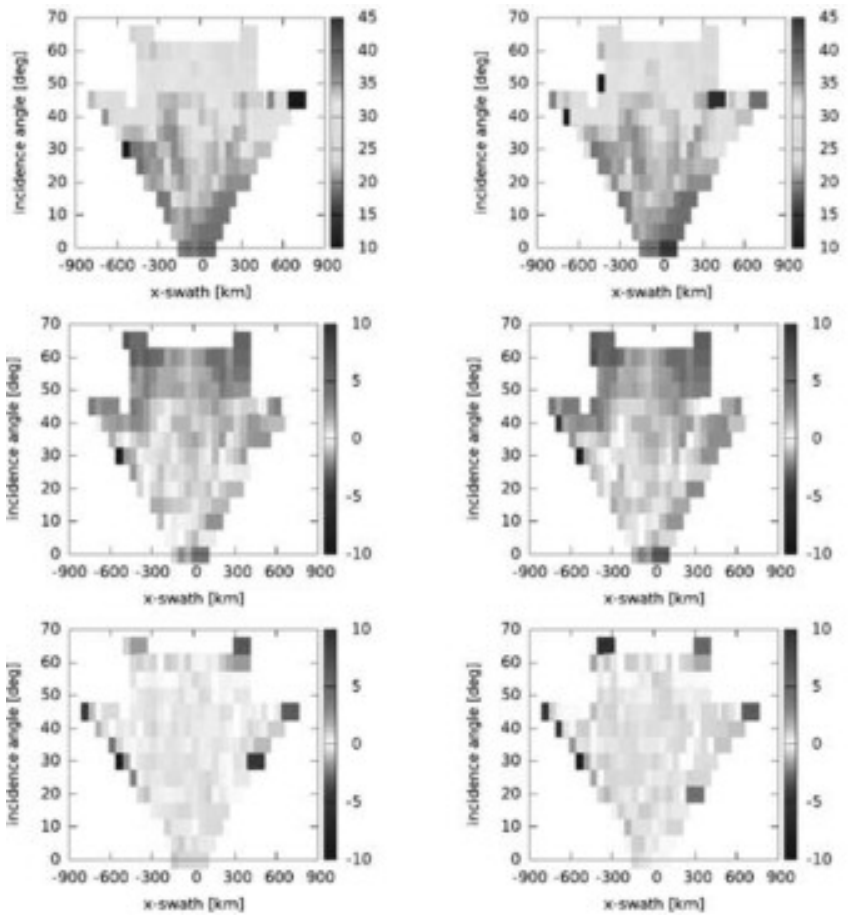
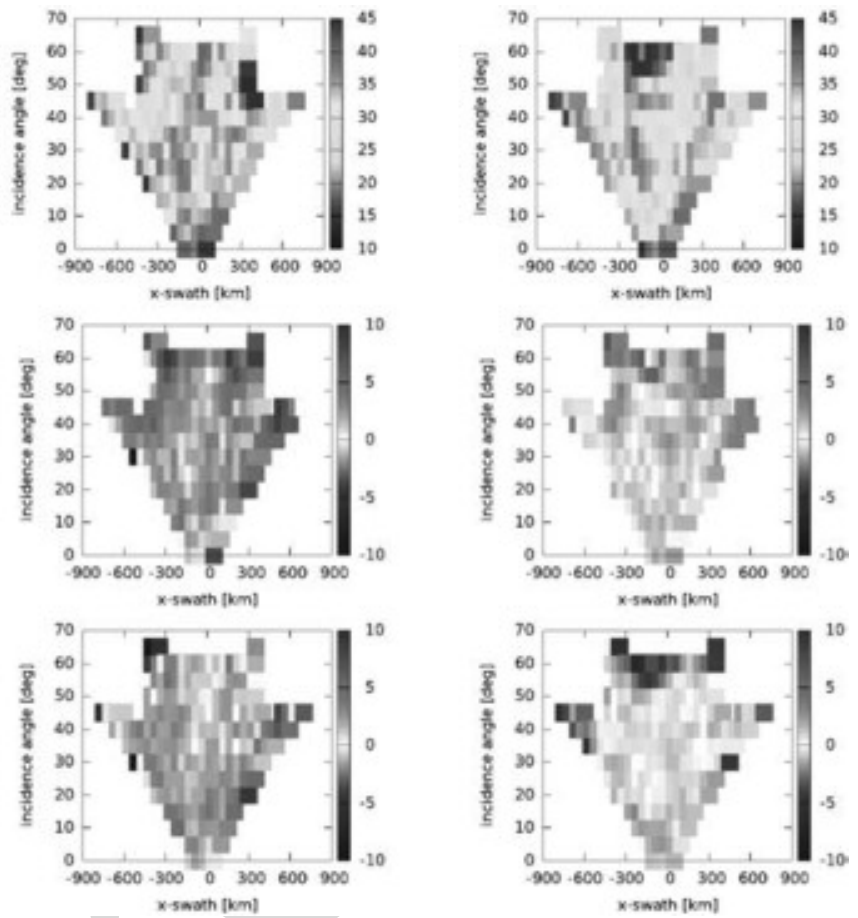

Fig. 1. SSS [psu] (first row) and TB biases [K] (computed following (3), second row) and differences between the empirical TB biases and the ones mitigated by OTT (third row) for four different points: (35S, 120W) in the Southern Pacific in the OTT region (first column); (30N, 140W) in the North Pacific (second column); (45N, 5E) in the Mediterranean Sea (third column); and (70N, 0E) in the Arctic Ocean (fourth column).

$$
\left\{\operatorname{SSS}_{\gamma}\right\} \equiv\{\operatorname{SSS}(\varphi, \lambda, d, x, \theta)\}
$$

each one referring to specific acquisition and geographical conditions. Note that, since $\gamma$ is time independent, we have essentially a time series of $\mathrm{SSS}_{\gamma}$ for each fixed set of $\gamma$ conditions.

The central estimator for each set $\left\{\mathrm{SSS}_{\gamma}\right\}$ (that we call SMOSbased climatology, $\operatorname{SSS}_{\gamma}^{c}$ ) represents a multiyear mean of the SSS value at that geographical location (determined by the longitude and latitude components of the tuple $\gamma$ ), together with the systematic bias associated with the position of the acquisition in the antenna (determined by $x$ and $\theta$ ) and the overpass direction $d$. Thus, the SMOS-based climatology $\operatorname{SSS}_{\gamma}^{c}$ can be used for correcting all the retrievals under the set of conditions comprised of $\gamma$. We use the central estimator proposed in [12]: the mean value of $\left\{\mathrm{SSS}_{\gamma}\right\}$ after discarding the outliers.

Once the geographical location and the overpass direction are fixed, the SMOS-based climatologies are represented in $(x, \theta)$ coordinates. This allows analyzing the structure of the errors depending on the position of the acquisition in the field of view. We call such a representation antenna climatology. In the first row of Fig. 1, the antenna climatologies of four different points are represented: (35S, 120W) in the Southern Pacific and lying in the OTT region; (30N, 140W) in the North Pacific; (45N, 5E) in the Mediterranean Sea; and (70N, 0E) in the Arctic Ocean. For the four cases, ascending satellite overpasses are considered. The first two antenna climatologies (open ocean) have similar values and patterns; in the last two plots (close to the coast and high latitudes, respectively) both mean values and patterns are markedly different. A change in the mean value is expected because the geophysical SSS climatologies of the four selected points are different. However, the observed differences provide evidence that the biases induced depend on the geographical distribution of the scene acquired. In particular, this implies that the OTT correction, which is based on assuming constant global errors, in the scene is in general not adequate when the presence of land or ice in the field of view changes the distribution of the TBs.

We convert each SMOS-based SSS climatology to an equivalent SMOS-based TB climatology as follows. We use the GMF for transforming the biases of SSS to TB biases, using the following relation:

$$
\mathrm{TB}_{\gamma}^{c}=\mathrm{TB}^{\mathrm{GMF}}\left(\theta, \operatorname{SSS}_{\gamma}^{c}, \mathrm{SST}^{\text {woa }}, \mathrm{WS}\right)
$$

where $\mathrm{TB}^{\mathrm{GMF}}$ is the modeled First Stokes parameter (according to the GMF), SST ${ }^{\text {woa }}$ is an annual climatological value of SST at the given geographical location $(\varphi, \lambda)$ provided by the World Ocean Atlas (WOA) 2013 (which is accessible at [17] and [18]), and WS is the wind speed and it has been fixed for all locations to $4 \mathrm{~m} / \mathrm{s}$.

Then, we estimate an antenna-climatology OTT correction at every point by

$$
\mathrm{OTT}_{\gamma}^{e}=\operatorname{TB}_{\gamma}^{c}-\operatorname{TB}^{\mathrm{GMF}}\left(\theta, \operatorname{SSS}^{\text {woa }}, \mathrm{SST}^{\text {woa }}, \mathrm{WS}\right)
$$

where $\mathrm{SSS}^{\mathrm{woa}}$ is the annual climatologies of SSS provided by the WOA 2013. Note that in (3), the contributions that are dependent on the wind (namely the roughness and galactic corrections) are cancelled out, because they equally contribute to both the $\mathrm{TB}_{\gamma}^{c}$ and the $\operatorname{TB}^{\mathrm{GMF}}\left(\theta, \mathrm{SSS}^{\text {woa }}, \mathrm{SST}^{\mathrm{woa}}, \mathrm{WS}\right)$ terms. Therefore, this analysis is independent of the fixed value of WS. In the second 

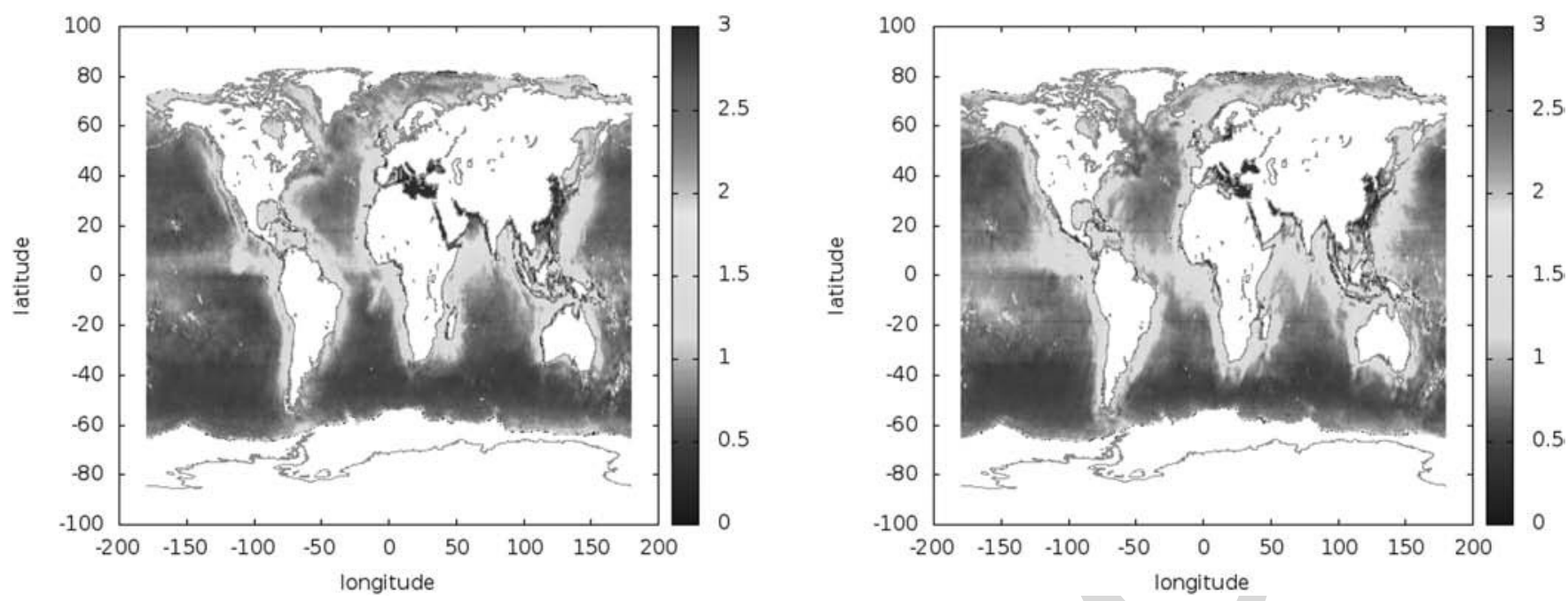

Fig. 2. Geographical distribution of the mean TB bias [K] metric computed following (5) for ascending (left) and descending (right) satellite overpasses.

row of Fig. 1, the OTT ${ }_{\gamma}^{e}$ is shown for the same four geographic points illustrated in the first row.

It is worth noting that the OTT bias correction depends on the time interval used in its computation. In the standard SMOS L2OS processor, ten days of data are used for the computation of a daily OTT. This implies that the OTT correction not only mitigates spatial biases, but also some other time-dependent biases. We are interested only in the component of error which is steady in time. Therefore, the results that we obtain with the antenna climatologies should be compared to a multiyear theoretical OTT. In fact, as the most significant variability of the OTT follows an annual cycle, it is sufficient to compute the average OTT over any full annual cycle. So, we use all the orbits in 2015 that cross $\Omega$ (the OTT region) to compute an annual average OTT $(\mathrm{OTT}(d, x, \theta))$. In the third row of Fig. 1, the differences between antenna-climatology and the classical OTTs for the four geographic points and ascending satellite overpasses are represented, according to the following expression:

$$
\Delta \operatorname{OTT}_{\varphi, \lambda, d}(x, \theta)=\operatorname{OTT}_{\gamma}^{e}-\operatorname{OTT}(d, x, \theta) .
$$

In the case of the two points in the open ocean (first and second plots), $\triangle \mathrm{OTT}$ is in general small, which means that the standard OTT correction allows mitigating the systematic biases at those regions to a good extent. However, differences in the Mediterranean Sea and in the Arctic Ocean are often larger than $5 \mathrm{~K}$ (in an absolute value) at many antenna positions. This means that OTT correction is not sufficient for removing systematic biases in these regions, and therefore other approaches are needed.

In order to have a global overview of the regions that are corrected well or poorly by the standard OTT, we define the following metric:

$$
\delta_{\mathrm{OTT}_{\varphi, \lambda, d}}=\frac{1}{N} \sum_{x, \theta}\left|\Delta \operatorname{OTT}_{\varphi, \lambda, d}(x, \theta)\right|
$$

where $N$ is the total number of $(x, \theta)$ points in the field of view. The two plots of Fig. 2 represent $\delta_{\mathrm{OTT}_{\varphi, \lambda, A}}$ (for ascending overpasses) and $\delta_{\mathrm{OTT}_{\varphi, \lambda, D}}$ (for descending overpasses). Coastal regions and high latitudes (especially the Arctic Ocean) are the regions where applying only the standard OTT correction is not sufficiently accurate.

\section{EMPIRICAL CHARACTERIZATION OF THE BRIGHTNESS TEMPERATURE UNCERTAINTY}

For a given acquisition condition and geographical location $\gamma$, the standard deviation of $\left\{\mathrm{SSS}_{\gamma}\right\}$, denoted by $\sigma_{\gamma}^{\text {SSS }}$, provides information about the multiyear variability of SMOS SSS. But $\sigma_{\gamma}^{\text {sSS }}$ contains both the geophysical variability and SMOSrelated variability. Once again, by using the GMF, we translate this SSS variability into TB variability as

$$
\sigma_{\gamma}^{\mathrm{TB}}=\sigma_{\gamma}^{\mathrm{SSS}} \cdot \partial_{\mathrm{SSS}} \mathrm{TB}^{\mathrm{GMF}}\left(\theta, \operatorname{SSS}_{\gamma}^{c}, \mathrm{SST}^{\text {woa }}, \mathrm{WS}\right)
$$

where $\partial_{\text {SSS }}$ notates the partial derivative with respect to the SSS

In Fig. $3, \sigma_{\gamma}^{\text {SSS }}$ (first row) and $\sigma_{\gamma}^{\mathrm{TB}}$ (second row) are represented in $(x, \theta)$ coordinates for the same four points in Fig. 1. As expected, $\sigma_{\gamma}^{\text {SSS }}$ (and similarly $\sigma_{\gamma}^{\mathrm{TB}}$ ) for the two open-ocean points are much smaller than for the points in the Mediterranean Sea and the Arctic Ocean. This is expected for two reasons: First, the geophysical variability of SSS is larger in the Mediterranean and in the Arctic Ocean than in the selected two open-ocean points; Second, the land-sea and ice-sea contaminations, and also RFI contaminations affecting the points in the Mediterranean and Arctic (respectively) lead to an increase in the respective uncertainties.

The different sources of variability in $\sigma_{\gamma}^{\mathrm{TB}}$ can be separated in the following terms:

$$
\left(\sigma_{\gamma}^{\mathrm{TB}}\right)^{2}=\left(\tilde{\sigma}_{\gamma}\right)^{2}+(\omega(\theta, \varphi, \lambda))^{2}+\left(\epsilon_{\gamma}\right)^{2}
$$

where $\tilde{\sigma}_{\gamma}$ represents the radiometric accuracy (that can be evaluated analytically, once the antenna temperature of the scene is known); $\omega^{2}$ represents the variability of TB induced by the geophysical variability of SSS, and $\epsilon$ is any residual or excess of uncertainty, that should be negligible in a perfect instrument 

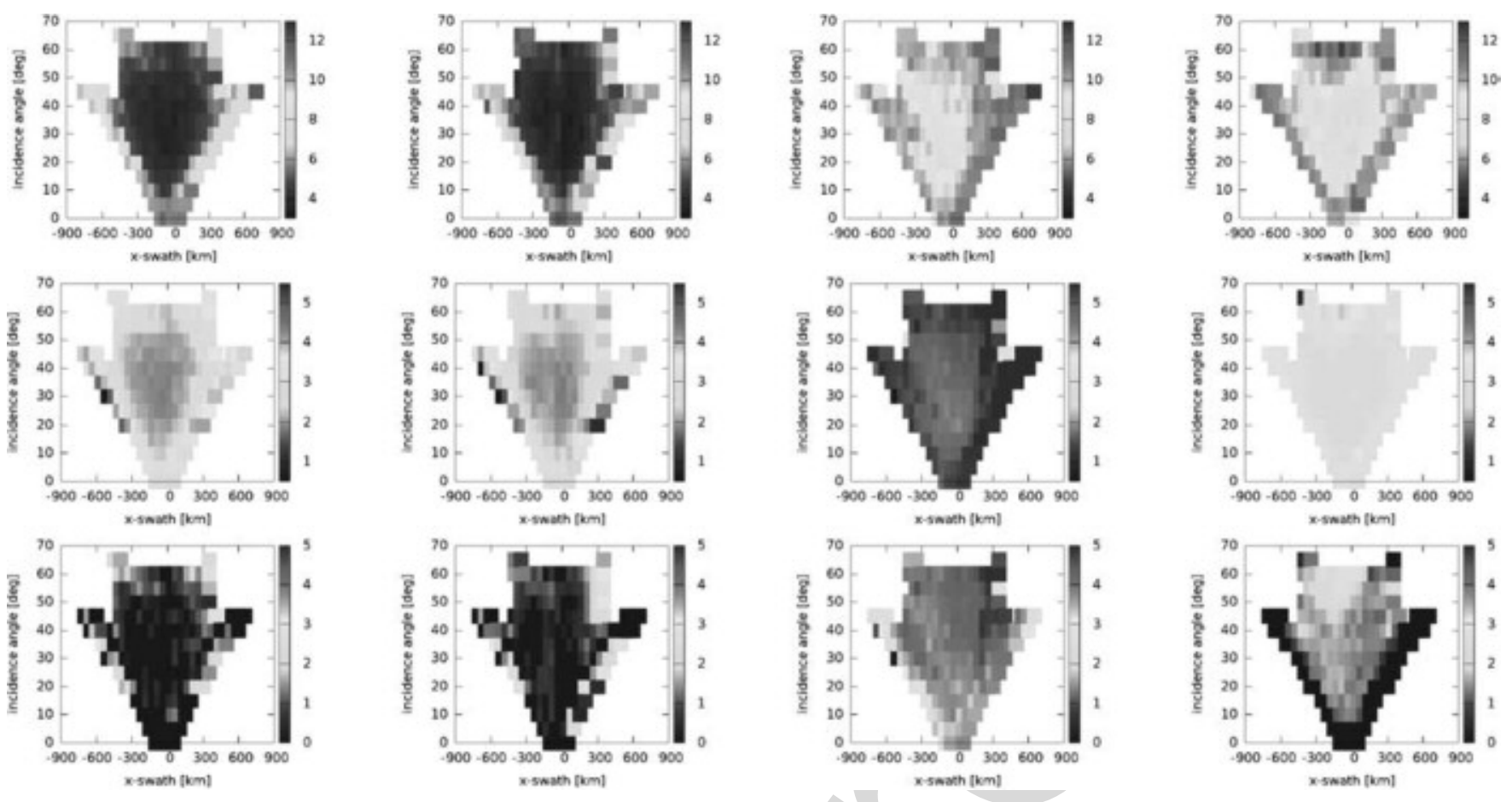

Fig. 3. SSS [psu] (first row) and TB [K] (second row) variability and the excess of TB uncertainty (third row) for four different points: (35S, 120W) in the Southern Pacific in the OTT region (first column); (30N, 140W) in the North Pacific (second column); (45N, 5E) in the Mediterranean Sea (third column); and $(70 \mathrm{~N}, 0 \mathrm{E})$ in the Arctic Ocean (fourth column). with no presence of RFI. We have approximated $\omega$ by

$$
\begin{aligned}
& \omega(\theta, \varphi, \lambda) \\
& =\omega_{\mathrm{SSS}}(\varphi, \lambda) * \partial_{\mathrm{SSS}} \mathrm{TB}^{\mathrm{GMF}}\left(\theta, \operatorname{SSS}^{\text {woa }}, \mathrm{SST}^{\text {woa }}, \mathrm{WS}\right)
\end{aligned}
$$

where $\omega_{\text {SSS }}$ has been computed as the standard deviation of the SMOS L3 SSS objectively analyzed nine-day maps provided by the Barcelona Expert Center (http://bec.icm.csic.es/oceanexperimental-dataset-global/) for the years 2011-2016 (the same time period used for the computation of $\sigma_{\gamma}^{\mathrm{TB}}$ ).

Then, the excess of uncertainty $\epsilon_{\gamma}$ can be computed by using (7). Plots in the third row of Fig. 3 represent the excess of uncertainty, $\epsilon_{\gamma}$ for the four points. In the case of the two openocean points, $\epsilon_{\gamma}$ is small and the only significant differences occur at the edges of the $(x, \theta)$ representation. However, in the Mediterranean Sea, the excess of uncertainty is greater than 3 $\mathrm{K}$ in almost the entire scene. Similarly, in the case of the Arctic Ocean point, there is an excess of uncertainty that is particularly large for incidence angles greater than $50^{\circ}$.

In order to estimate the magnitude of $\epsilon$ at each location, we define the following metric:

$$
\delta \epsilon_{\varphi, \lambda, d}=\sqrt{\frac{1}{N} \sum_{x, \theta}\left(\epsilon_{\gamma}\right)^{2}}
$$

where $N$ is the number of $(x, \theta)$ points in the field of view. Fig. 4 shows two maps of $\delta \epsilon$ : the left one for ascending orbits and the right one for descending orbits. The following conclusions can be drawn.

1) An excess of $\mathrm{TB}$ uncertainty is expected in regions strongly affected by RFI, as, for example, in the Chinese and Arabian Seas, Bay of Bengal, close to Madagascar, or in the European semi-enclosed Seas (Mediterranean Sea and Black Sea).

2) However, we also observe an increase of the excess of uncertainty close to the land-sea transitions (coastal areas) and also close to ice-sea transitions (mainly in the Arctic Ocean). Both land-sea and ice-sea contaminations have been treated until now (in the official ESA L2OS processor) as an increase in the bias, but not in the uncertainty. The increase of the uncertainty close to the coast could be explained by the variability of land surface TB, which may contaminate the field of view. The orientation of the antenna footprint could also produce an increase in the uncertainty close to the coast.

\section{ANALYsis OF THE THIRD- AND FOURTH-ORDER MOMENTS OF THE SMOS-BASED CliMATOLOGY DisTRIBUTIONS}

For fixed acquisition conditions $\gamma$, the skewness and the kurtosis of the $\left\{\mathrm{SSS}_{\gamma}\right\}, \mathrm{ske}_{\gamma}^{\mathrm{SSS}}$ and $\mathrm{kur}_{\gamma}^{\mathrm{SSS}}$, respectively, provide a measure of the symmetry or asymmetry and flatness or peakedness, respectively, of the SMOS-based climatological salinity distributions associated with $\left\{\mathrm{SSS}_{\gamma}\right\}$. They are computed as follows:

$$
\begin{aligned}
\operatorname{ske}_{\gamma}^{\mathrm{SSS}} & =\frac{E\left[\mathrm{SSS}_{\gamma}-\mu\right]^{3}}{\left(E\left[\mathrm{SSS}_{\gamma}-\mu\right]^{2}\right)^{3 / 2}} \\
\operatorname{kur}_{\gamma}^{\mathrm{SSS}} & =\frac{E\left[\mathrm{SSS}_{\gamma}-\mu\right]^{4}}{\left(E\left[\mathrm{SSS}_{\gamma}-\mu\right]^{2}\right)^{2}}
\end{aligned}
$$



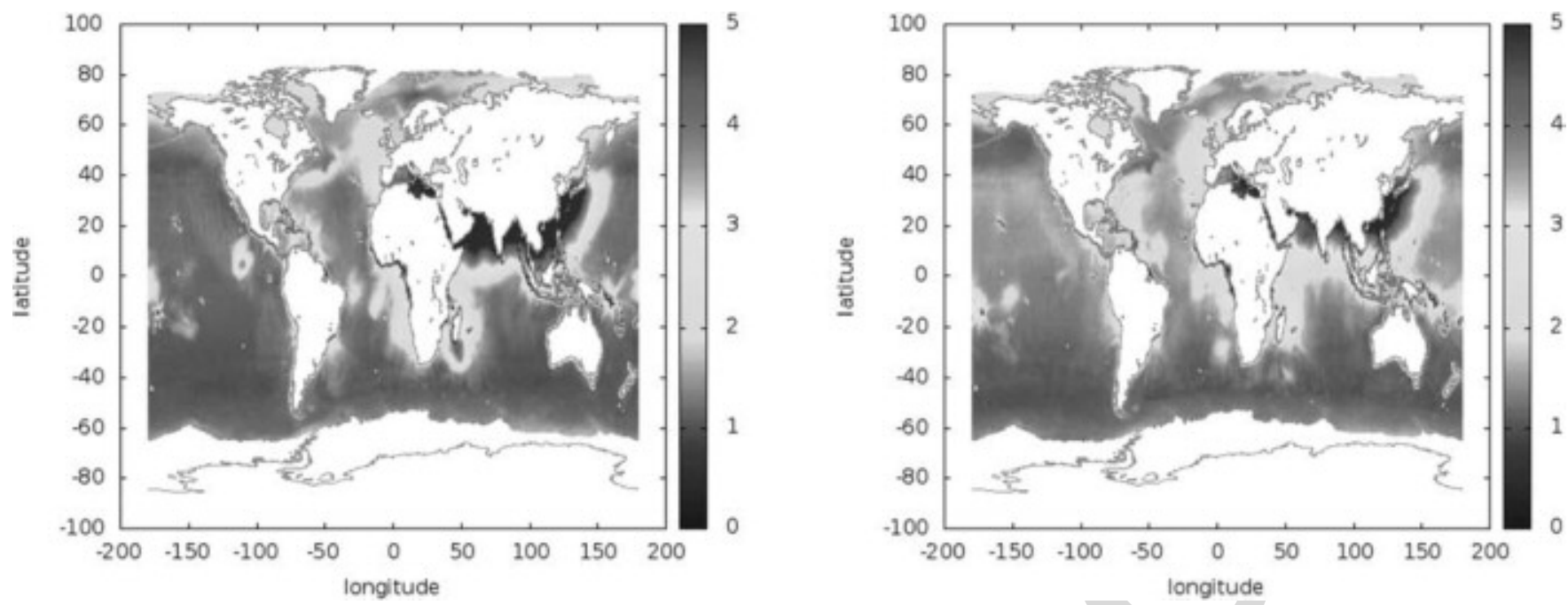

Fig. 4. Geographical distribution of the excess of TB uncertainty [K] for ascending (left) and descending (right) satellite overpasses.
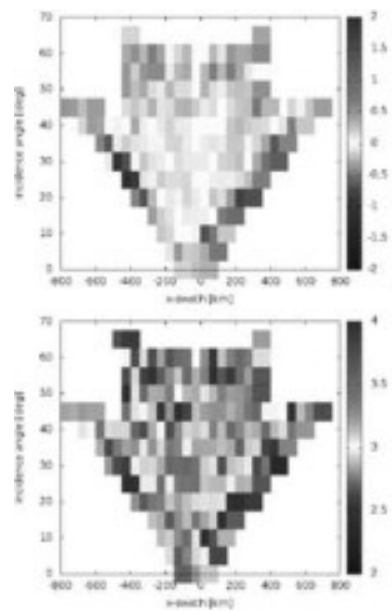
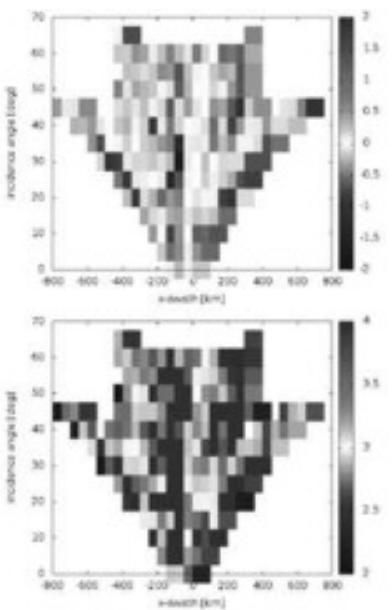
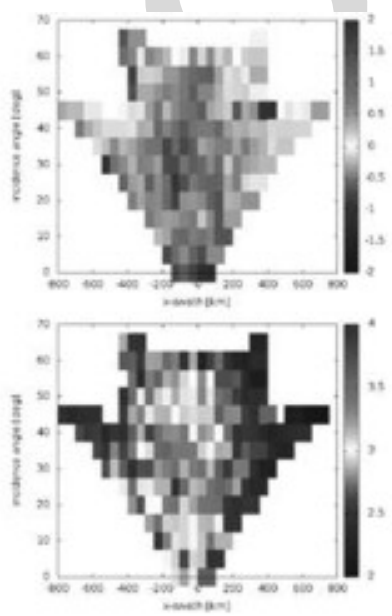
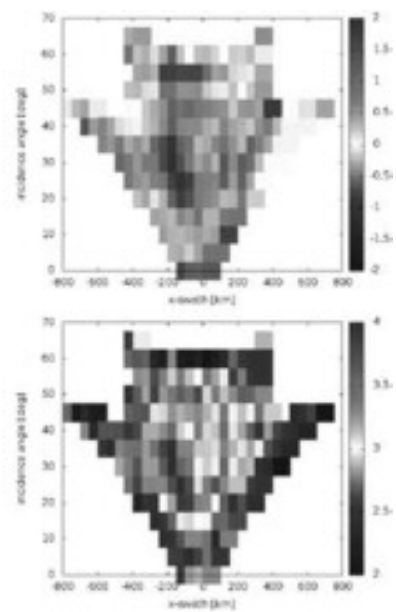

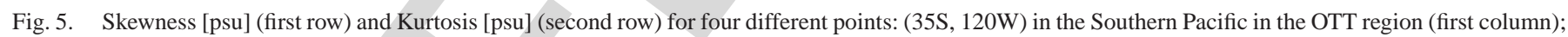
$(30 \mathrm{~N}, 140 \mathrm{~W})$ in the North Pacific (second column); (45N, 5E) in the Mediterranean Sea (third column); and (70N, 0E) in the Arctic Ocean (fourth column).

where $E[]$ is the expectation operator and $\mu$ is the mean of $\left\{\mathrm{SSS}_{\gamma}\right\}$. In the first row of Fig. 5, ske $\mathrm{SSS}_{\gamma}$ is represented in $(x, \theta)$ coordinates for the same four points considered previously. Absolute values of skewness lower than 0.5 correspond to rather symmetric distributions, while larger values imply that the distributions are significantly skewed either to the right (positive skewness) or to the left (negative skewness).

The absolute value of the $\mathrm{ske}_{\gamma}^{\mathrm{SSS}}$ for the point located in the OTT region is almost everywhere lower than 0.5 , except for the points near the edge of the swath, which are higher. This indicates that for this point the distributions are mainly symmetric across the majority of the swath. The skewness for the additional open-ocean point (North Pacific) is not so homogeneous as for the point in the OTT region. In this case, in different positions of the swath, different values of skewness are observed, some of them larger than 0.5 and others smaller than -0.5 . In particular, for a fixed across-track distance value, one can find positive and negative skewness values (asymmetric distributions). In the case of the other two points (Mediterranean Sea and Arctic Ocean) the corresponding skewness values are mainly lower than -0.5 , what indicates that the distributions are asymmetric for the majority of the field of view, and most often skewed to the left.

In the second row of Fig. 5, kur ${ }_{\gamma}^{\text {SSS }}$ is represented in $(x, \theta)$ coordinates for the same four points. Kurtosis values larger than 3 indicate peaked distributions while kurtosis values lower than 3 indicate flat distributions. The two points in the open ocean display values of kurtosis close to 3 (i.e., close to Gaussian distribution) or greater than 3 (i.e., peaked distributions). This implies that for these coordinates, the central estimators (the SMOSbased climatology) are well-defined. For the two other points (in the Mediterranean Sea and in the Arctic Ocean) there are zones in the antenna where the kurtosis is close to or greater than 3 (and therefore they correspond to peaked distributions) but at other zones the kurtosis is significantly lower than 3 , implying flatter distributions and hence a poorly defined central estimator. 

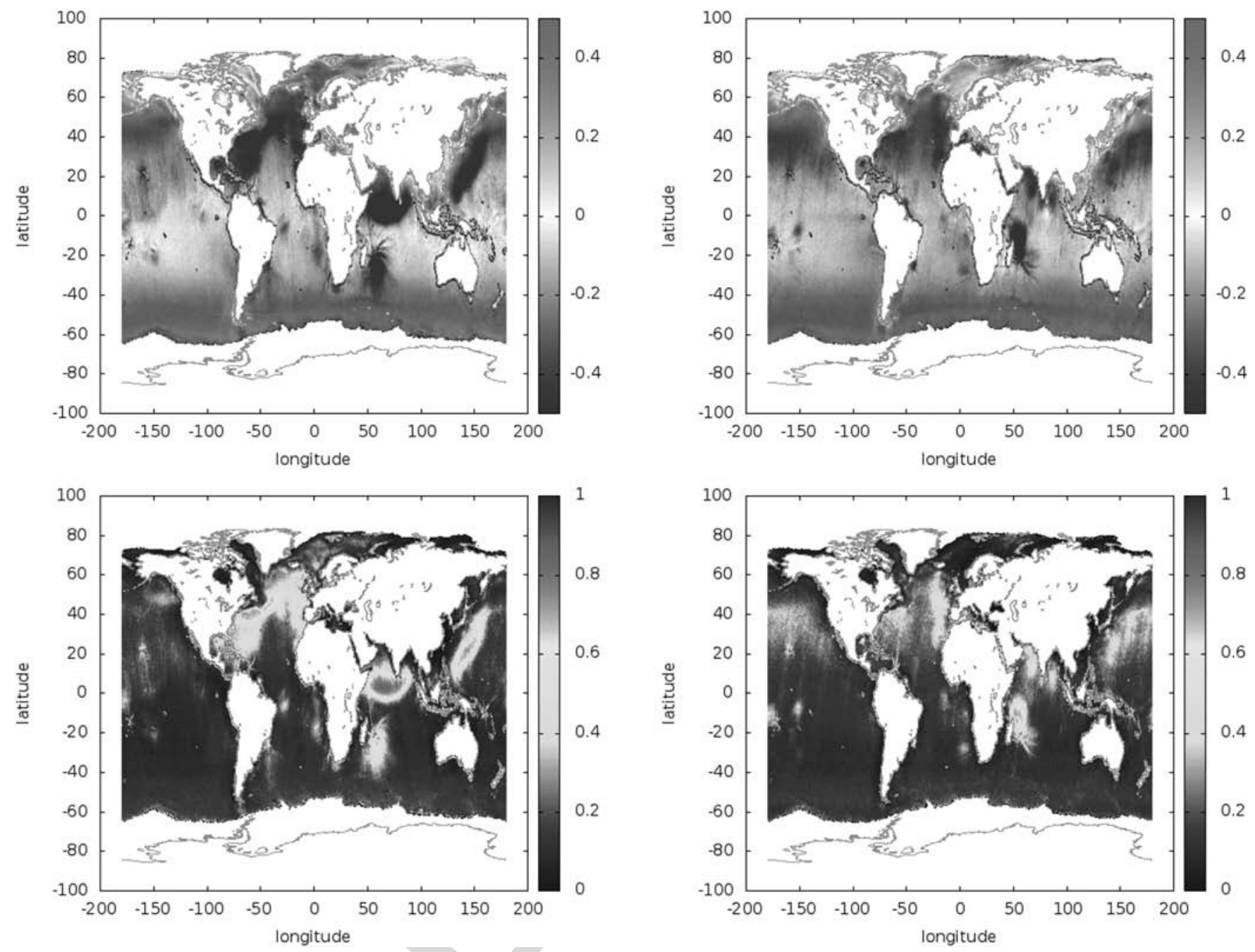

Fig. 6. Geographical distribution of the mean value of the skewness ([psu]) (top) and probability of skewness larger than 0.5 [psu] in the absolute value $\left(\delta\right.$ ske $_{\varphi, \lambda, d}$ as defined in 12): left ascending overpasses; and right descending satellite overpasses.

In order to identify those regions with greater asymmetry, we define the probability of a given location having SSS distributions with an absolute value of skewness larger than 0.5 as

$$
\delta \operatorname{ske}_{\varphi, \lambda, d}=\frac{1}{N} \sum_{x, \theta}\left(\delta_{\text {ske }}\right)
$$

where $N$ is the number of $(x, \theta)$ points that have been taken into account and $\delta_{\text {ske }}$ is defined as follows:

$$
\delta_{\text {ske }}=\left\{\begin{array}{ll}
1, & \left|\operatorname{ske}_{\gamma}^{\mathrm{SSS}}(\varphi, \lambda, d, x, \theta)\right|>0.5 \\
0, & \left|\operatorname{ske}_{\gamma}^{\mathrm{SSS}}(\varphi, \lambda, d, x, \theta)\right| \leq 0.5
\end{array} .\right.
$$

Fig. 6 shows four maps. In the first row, the mean value of ske $_{\gamma}^{\mathrm{SSS}}$ in the antenna climatology is represented at each location for ascending (left) and descending (right) orbits. In the full ocean, the mean skewness value in the antenna climatology is mainly negative. The skewness becomes more negative in those regions that are especially affected by RFI such as the Northern Indian Ocean, the Chinese Sea, the North Atlantic European coast or near Madagascar and also in the Southern Ocean. Regions with very low SSS values, such as the Black and the Baltic seas have in general positive skewness because the climatological distributions are truncated. Only positive SSS values (and lower than $50 \mathrm{psu}$ ) are considered in the climatological distributions. In both the Black and Baltic Seas the mean SSS values are typically below $20 \mathrm{psu}$. Since the corresponding $\sigma_{\gamma}^{\mathrm{SSS}}$ are also large (close to $10 \mathrm{psu}$ ), the negative tail of those distributions is truncated and this results in positive skewness values. In the second row, maps representing $\delta \operatorname{ske}_{\varphi, \lambda, d}$ are shown: one for ascending orbits (left) and the other one for descending orbits (right), indicating those regions with symmetric/a symmetric distributions.

Moreover, in order to identify the regions with flat distributions (and therefore, problematic regions), we define the probability of a given location having SSS distributions with kurtosis values lower than 3 as

$$
\delta \operatorname{kur}_{\varphi, \lambda, d}=\frac{1}{N} \sum_{x, \theta}\left(\delta_{\text {kur }}\right)
$$



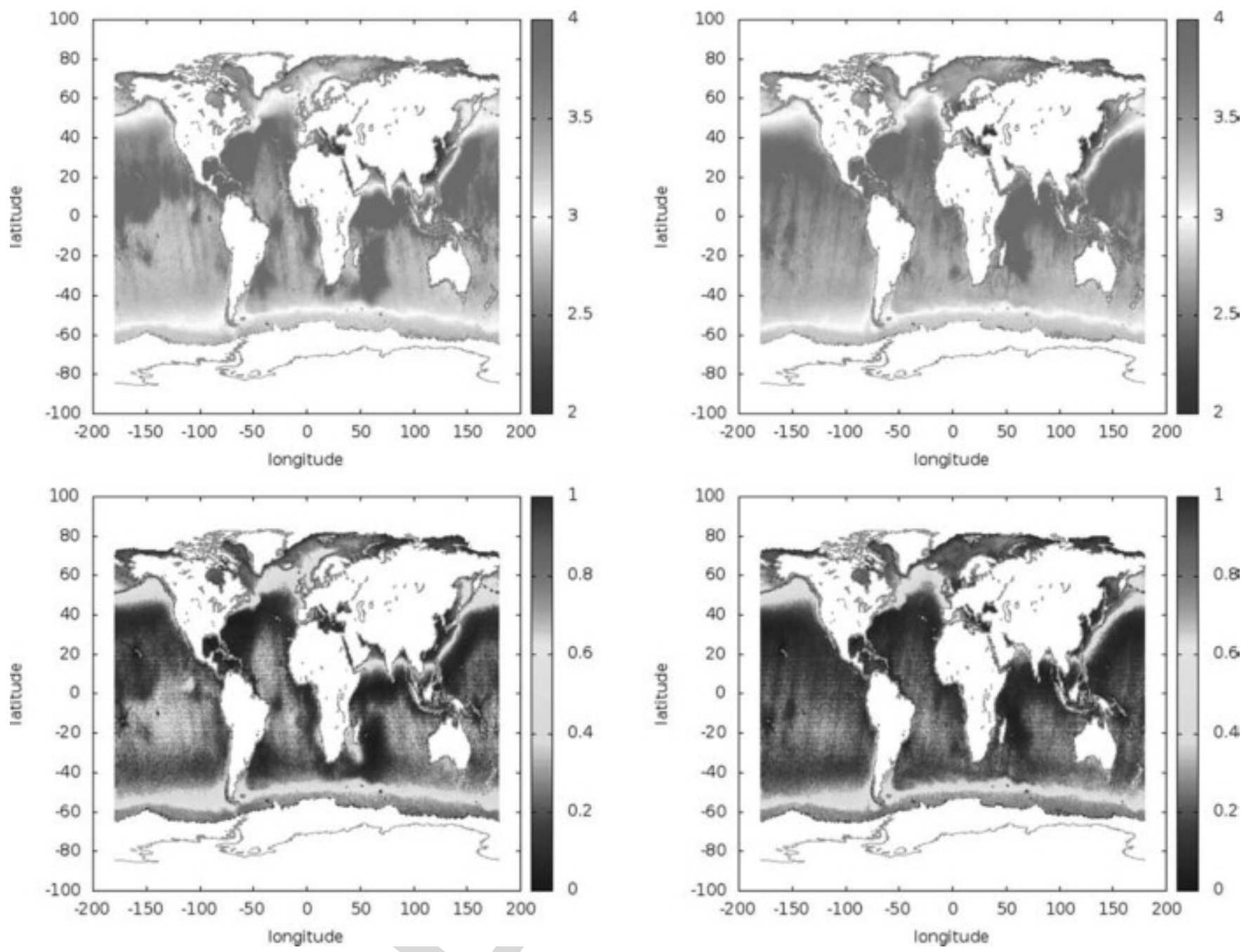

Fig. 7. Geographical distribution of the mean value of the kurtosis ([psu]) (top) and probability of the kurtosis value being lower than 3 [psu] ( $\delta \mathrm{kur}_{\varphi, \lambda, d}$ as defined in 14): left ascending overpass; and right descending satellite overpass.

where $N$ is the number of $(x, \theta)$ points in the antenna and $\delta_{\text {kur }}$ is defined as follows:

$$
\delta_{\text {kur }}=\left\{\begin{array}{ll}
1, & \operatorname{kur}_{\gamma}^{\mathrm{SSS}}(\varphi, \lambda, d, x, \theta)<3 \\
0, & \operatorname{kur}_{\gamma}^{\mathrm{SSS}}(\varphi, \lambda, d, x, \theta) \geq 3
\end{array} .\right.
$$

In the first row of Fig. 7, the mean value of $\operatorname{kur}_{\gamma}^{\mathrm{SSS}}$ in the antenna climatology is represented at each location for ascending (left) and descending (right). The behavior of the kurtosis follows a latitudinal trend. At medium and low latitudes, the distributions are peaked but at high latitudes the kurtosis decreases. Near the European and Asian coasts, the distributions also have values of kurtosis lower than 3. In the second row of Fig. 7, $\delta \operatorname{kur}_{\varphi, \lambda, d}$ is shown for ascending orbits (left) and descending orbits (right).

By considering the skewness and the kurtosis together, we can define a unique metric for determining which locations have acceptable distributions, in terms of the reliability of the SMOSbased climatology, essentially, we look for rather symmetric, reasonably peaked distributions. Our metric is defined as

$$
\delta \operatorname{Dis}_{\varphi, \lambda, d}=\frac{1}{N} \sum_{x, \theta}\left(\delta_{\mathrm{dis}}\right)
$$

where $N$ is the number of $(x, \theta)$ points in the antenna and $\delta_{\text {dis }}$ is defined as follows:

$$
\delta_{\text {dis }}=\max \left\{\delta_{\text {ske }}, \delta_{\text {kur }}\right\}
$$

Fig. 8 shows two maps of $\delta \operatorname{Dis}_{\varphi, \lambda, d}$ : one for ascending orbits (left) and the other one for descending orbits (right). Along the European and Asian coasts the distributions are degraded.

The eclipse and some sources of RFI [7] degrade the quality of the measurements. The eclipse periods are from mid-November to mid-February. During an eclipse, the Sun is occulted by the earth, which produce a sudden decrease of the antenna skin temperature of a couple of tens of degrees (from around $28{ }^{\circ} \mathrm{C}$ to some $5{ }^{\circ} \mathrm{C}$ ). When the satellite exits the earth shadow, the Sun warms up the antenna again until it reaches the temperature it
420 421 

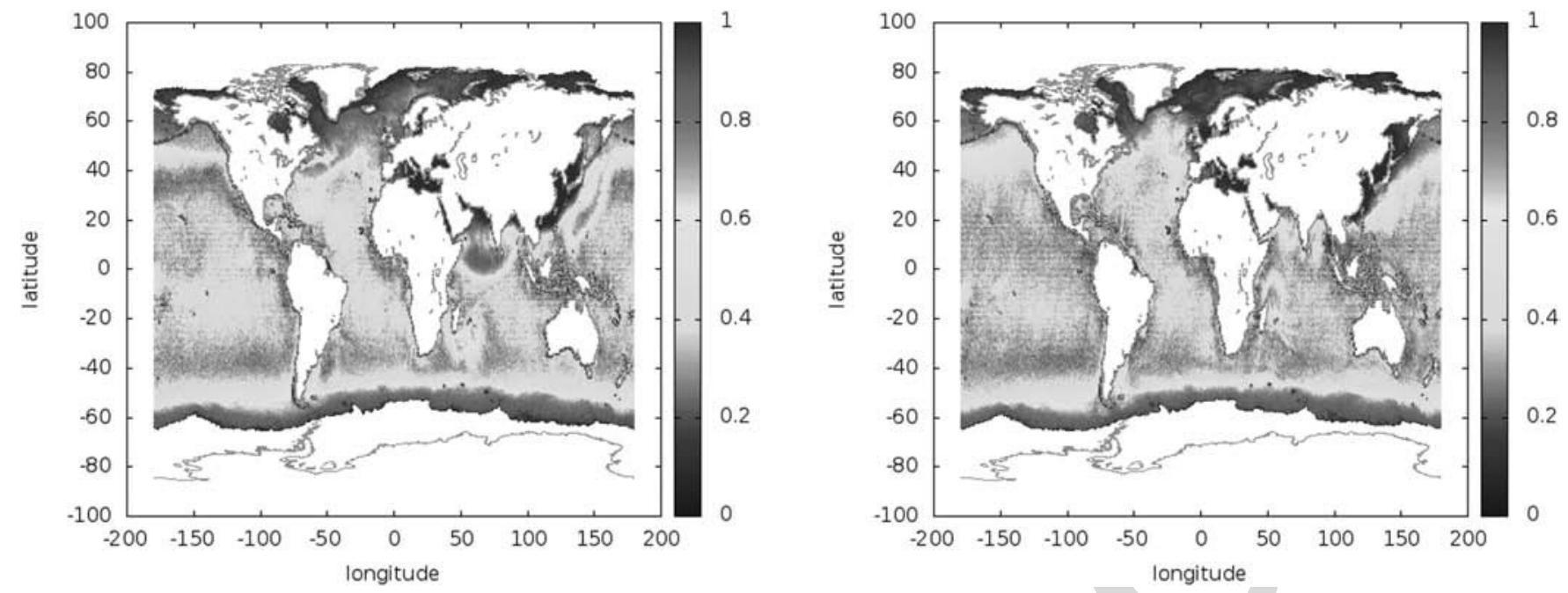

Fig. 8. Geographical distribution of $\delta \operatorname{Dis}_{\varphi, \lambda, d}$ for ascending (left) and descending (right) satellite overpass. $\delta$ Dis $\varphi_{\varphi, \lambda, d}$ values provide a metric of the locations where distributions $\left\{\operatorname{SSS}_{\gamma}\right\}$ are less accurate: the largest the value of $\delta \operatorname{Dis}_{\varphi, \lambda, d}$; the largest the number of poorly conditioned distributions due to flatness or asymmetry.

would have if there had been no eclipse. This post-eclipse transient causes, in every descending orbit, a warm anomaly in the TB, which extends to latitudes as low as $30^{\circ}$ North [7]. These effects are not permanent in time, and therefore, they may lead to asymmetric distributions. The main sources of TB errors (both RFI and sea-ice and LSC) artificially increase the TB values, producing negative biases in SSS, which could explain the negative skewness shown in the first row of Fig. 6. Additionally, in coastal regions affected by RFI (such as the European and Asian coasts) the number of retrievals typically decreases. The reduction of retrievals together with an increase of the uncertainty (see Fig. 4) could also lead to flatter distributions, i.e., distributions with low values of kurtosis.

On the other hand, at high latitudes (Arctic and Antarctic Oceans), the distributions are also degraded. This could be an effect of the latitudinal and seasonal bias. Moreover, the sensitivity of the $T_{B}$ to salinity decreases in cold waters [19]. As shown in [20], such sensitivity drops from $0.5 \mathrm{~K} /$ psu to $0.3 \mathrm{~K} / \mathrm{psu}$ when SST decreases from $15^{\circ} \mathrm{C}$ to $5^{\circ} \mathrm{C}$. This loss of sensitivity could lead to flatter distributions with kurtosis lower than 3 . This could explain the blue regions at high latitudes in the plots on the first row of Fig. 7. These results seem to indicate that those regions where distributions are degraded in terms of symmetry and flatness are those affected by the latitudinal and seasonal biases and RFI.

\section{DEFINITION OF A LEVEL 2 SMOS SSS PRODUCT}

The characterization of the empirical biases and uncertainties analyzed in the two previous sections are used for the generation of an alternative SMOS Level 2 product. The non-Bayesian single angle retrievals belonging to the set $\left\{\mathrm{SSS}_{\gamma}\right\}$ are corrected with their corresponding $\mathrm{SSS}_{\gamma}^{c}$ for removing systematic spatial biases (SMOS-based anomalies). Debiased non-Bayesian retrievals are subsequently obtained after adding the annual WOA2013 SSS reference to the SMOS-based anomalies. We use the methodology in [12] to generate SMOS Level 3 SSS maps and the following improvements. On the one hand, we propose a new Bayesian scheme that accounts for the uncertainties studied in Section III: the posterior-Bayesian (hereafter post-Bayesian) retrieval. To generate the post-Bayesian L2 SSS, all debiased non-Bayesian retrievals corresponding to the same dwell line are averaged and weighted by its respective SMOSbased SSS variance $\left(\sigma_{\gamma}^{\text {SSS }}\right)^{2}$. On the other hand, the filtering criteria proposed by [12] are modified in order to be more consistent with the estimated uncertainty, and to better preserve the SSS gradients measured by SMOS. Thus, the following four filtering criteria are applied to the SSS.

1) SMOS-based climatological filters: For given acquisition conditions $\gamma$, the set of all the $\mathrm{SSS}$ belonging to $\left\{\mathrm{SSS}_{\gamma}\right\}$ are discarded when one of the following conditions is satisfied.

a) Standard deviation $\left(\sigma_{\gamma}^{\mathrm{SSS}}\right)$ is greater than $10 \mathrm{psu}$.

b) The set of $\sigma_{\gamma}^{\text {SSS }}$ contains less than $100 \mathrm{SSS}$ measurements.

c) The absolute value of its skewness is greater than $1 \mathrm{psu}$.

d) Its kurtosis is lower than 2 psu.

2) Outlier with respect to its $S S S_{\gamma}^{c}$ : At this step, we distinguish between symmetric and peaked distributions and asymmetric and flat distributions. So, when $\mid$ ske $_{\gamma}^{\mathrm{SSS}} \mid \leq 0.25$ and $\operatorname{kur}_{\gamma}^{\mathrm{SSS}}>2.5$, we discard the SSS belonging to $\left\{\mathrm{SSS}_{\gamma}\right\}$ such that

$$
\left|\mathrm{SSS}-\mathrm{SSS}_{\gamma}^{c}\right|>2 \sigma_{\gamma}^{\mathrm{SSS}}
$$

otherwise, we discard the SSS such that

$$
\left|\mathrm{SSS}-\mathrm{SSS}_{\gamma}^{c}\right|>\sigma_{\gamma}^{\mathrm{SSS}}
$$

In any case, we always discard SSS such that

$$
\left|\mathrm{SSS}-\operatorname{SSS}_{\gamma}^{c}\right|>10 \text { psu. }
$$


3) Outlier with respect to the SSS values of the same dwell line: After the spatial bias correction, we consider a cylindrical grid of $0.25 \times 0.25^{\circ}$ for the daily L2 product generation [21]. Then, we compute the mean $\bar{s}_{L 2}$ and the standard deviation $\sigma s_{L 2}$ of the set of debiased SSS that belongs to a given dwell line, $s_{L 2}$. We use $\sigma s_{L 2}$ to filter out outliers: we discard the SSS of $s_{L 2}$ such that $\left|s_{L 2}-\bar{s}_{L 2}\right|<\sigma s_{L 2}$.

4) All the points (in the $0.25 \times 0.25^{\circ}$ grid) with less than 5 valid SSS are discarded.

The need for an additional time correction in this methodology is described in [12]. The applied debiasing technique does not depend on time, while the OTT correction is applied every day. That suggests that the SSS retrieval requires not only a spatial bias mitigation, but also a temporal bias mitigation. Different approaches were studied in [12], and the authors finally proposed removing the mean value of the SMOS-based anomalies corresponding to each nine-day map. Here, using the same idea, we remove the mean value of the SMOS-based anomalies corresponding to each daily map. While in [12], the L3 maps are computed by using ascending and descending orbits together; here, the daily maps are computed separately for ascending and descending satellite overpasses. Using a temporal correction based on daily maps and not on nine-day maps causes an increase in the noise with respect to the correction proposed in [12].

\section{AssesSMent OF THE SMOS SSS LeVEL 2 PRODUCTS}

In this section, we compare the following SMOS SSS Level 2 products:

1) Unfiltered SSS v662: The first SSS field provided in the last version (v662) of the L2 SMOS SSS User Data Product (UDP) is considered. This field is distributed with an empirical LSC correction applied. Therefore, nonsignificant biases close to the coasts are expected.

2) SSS v662: Usually, the SSS values provided by the UDP are filtered before being used in the computation of L3 maps. We have used the filtering criteria applied in the generation of the operational SMOS SSS product distributed by the Barcelona Expert Centre (see [22] for more details). Note that the filtered L2 v662 consists of a posteriori filtering on the retrieved L2 SSS, i.e., both the unfiltered and the filtered L2 SSS products are identical in those grid points where the latter has valid SSS data.

3) SSS post-Bayesian: SSS computed as explained in Section V.

One year (2016) of daily maps at $0.25 \times 0.25^{\circ}$ resolution on a cylindrical grid has been generated for each one of the three previous L2 products, and compared to Argo float data to assess the quality.

\section{A. Quality Assessment Approach}

A statistical comparison between daily SMOS SSS maps and the uppermost near surface salinity values acquired by Argo floats is carried out for 2016. Argo data are freely distributed by CORIOLIS, http://www.coriolis.eu.org. The cutoff depth for Argo profiles is taken at $10 \mathrm{~m}$, but no measurements shallower than $0.5 \mathrm{~m}$ are used due to the formation of bubbles and foam. In the case of SOLO and PROVOR Argo floats, only the data deeper than $5 \mathrm{~m}$ are used because their conductivity, temperature, and depth probes stop pumping water at around 5-m below the surface [23]. Profiles from BioArgo and those included in the graylist (i.e., floats that may have problems with one or more sensors) are discarded. In addition, we use WOA 2013 as an indicator: Argo float profiles with anomalies larger than $10^{\circ} \mathrm{C}$ in the temperature or $5 \mathrm{psu}$ in salinity when compared to WOA are discarded. Only profiles having a temperature close to surface between -2.5 and $40{ }^{\circ} \mathrm{C}$ and a salinity between 2 and 41 psu are used.

\section{B. Coverage of the SMOS SSS L2 Products}

In Fig. 9, regional daily maps (August 3rd, 2016) showing the North Atlantic, the Mediterranean Sea, and part of the Arctic Ocean are presented for the three L2 products under study: unfiltered SSS v662 (top); SSS v662 (middle); and post-Bayesian SSS (bottom).

In the Mediterranean and the Northern seas, the unfiltered SSS v662 (top) presents many artifacts with SSS values that are not geophysical (they are too fresh). So, the application of filters in these regions is mandatory. However, after the application of the filters (middle plot), the number of valid retrievals dramatically decreases: few valid measurements are obtained near the European coast, in the Mediterranean Sea; and the Gulf of Stream. The post-Bayesian product (bottom plot) noticeably improves the coverage, as compared to the filtered L2 v662, and more valid SSS retrievals in the Mediterranean Sea, the Norwegian Sea, and in the Arctic ocean are now obtained.

Close to the coast, the filtered v662 seems too restrictive. The unfiltered product provides SSS values in the vicinity of the land grid points. Coastal pixel values in the unfiltered v662 are not geophysically reasonable, which suggests a residual LSC are typically fresher than $32 \mathrm{psu}$. The LSC is typically present as a negative bias in the SMOS SSS. The coastal pixel values lower than 32 psu in the non-filtered v662 are not geophysically reasonable, which suggests that a residual LSC bias could still affect the most coastal pixels of this product. The post-Bayesian product also yields valid SSS data in grid points close to land. In this case, the salinities are not as fresh (with more geophysical significance) as the ones shown by the unfiltered product, which suggests that the LSC is better mitigated when using the postBayesian methodology. These plots suggest that a residual LSC bias could still affect the most coastal pixels of the v662 product.

\section{Comparison With Daily Argo Salinity}

Table I describes the statistics corresponding to the following three regions:

1) global ocean (Global): between $60^{\circ} \mathrm{S}$ and $60^{\circ} \mathrm{N}$ (all longitudes);

2) northern ocean (NOC): between $40^{\circ} \mathrm{N}$ and $60^{\circ} \mathrm{N}$ (all longitudes);

3) southern ocean (SOC): between $60^{\circ} \mathrm{S}$ and $40^{\circ} \mathrm{S}$ (all longitudes). 


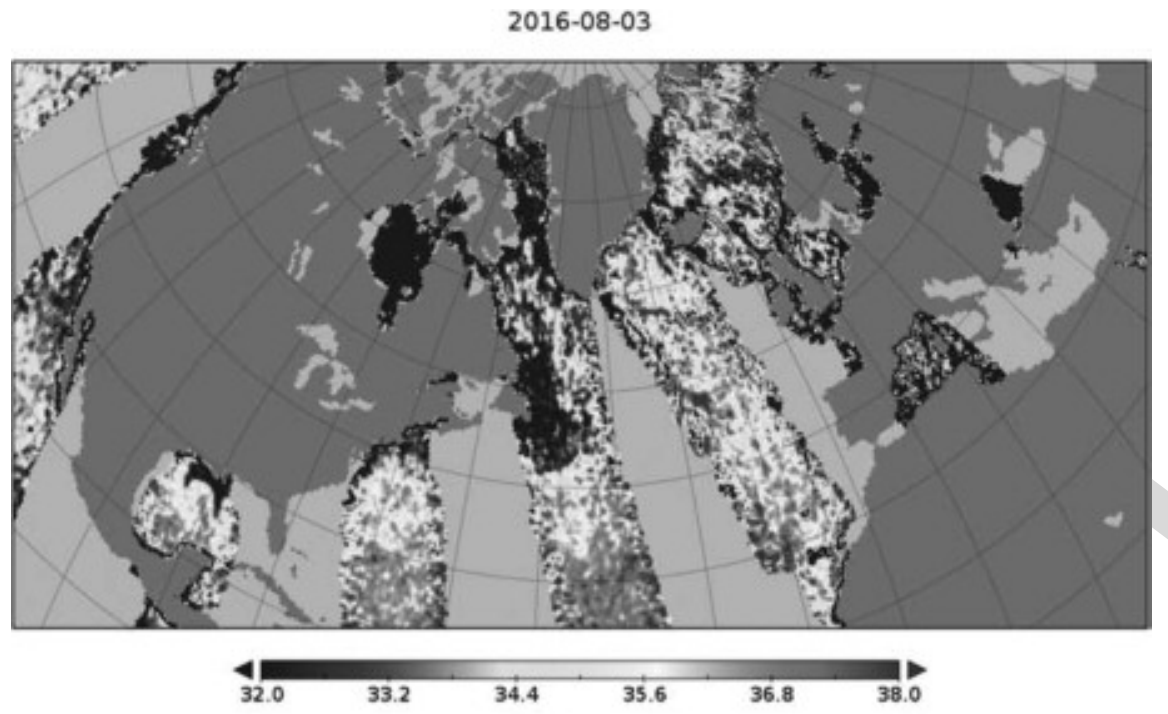

(a)

2016-08-03

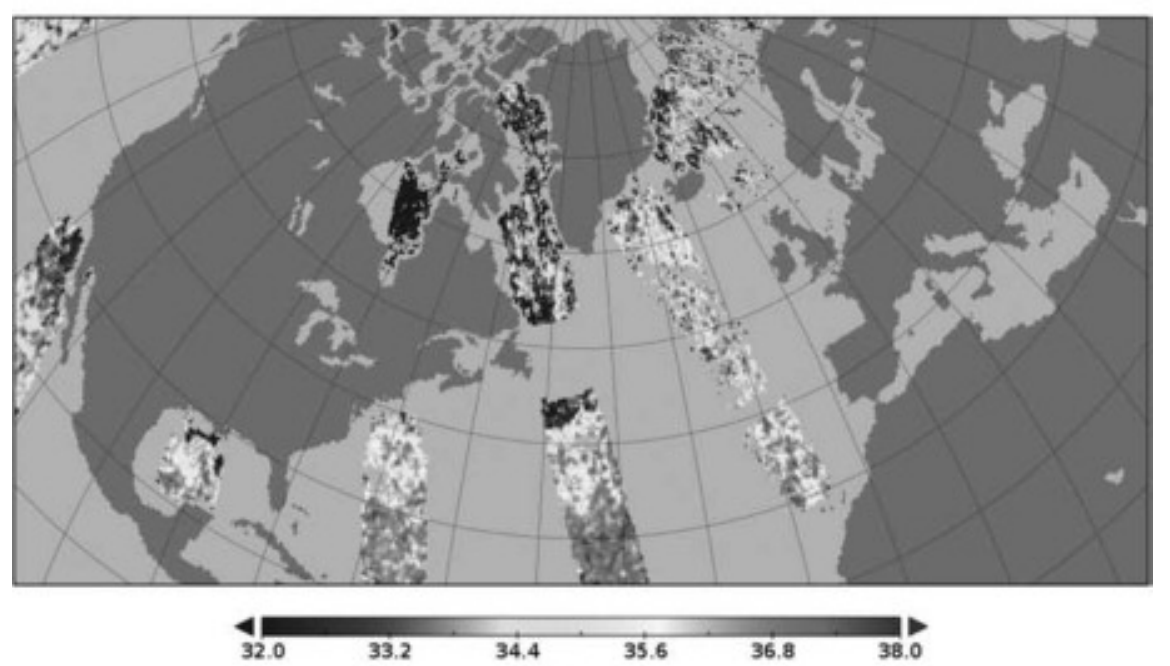

(b)

2016-08-03
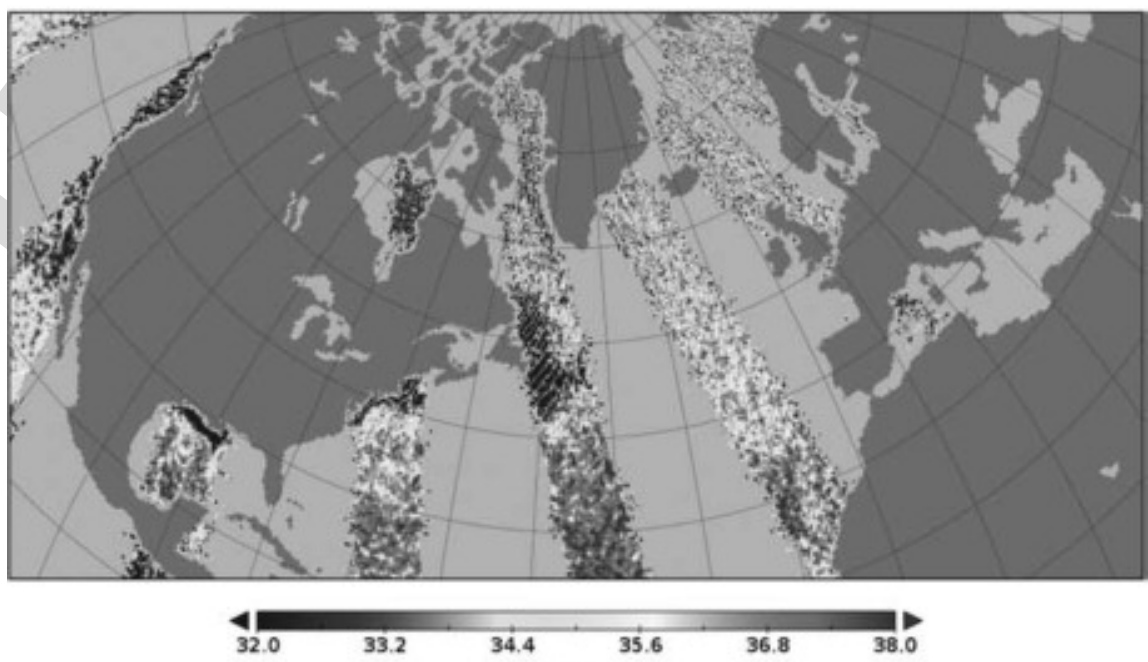

(c)

Fig. 9. SMOS L2 SSS products. (a) Unfiltered v662. (b) v662. (c) Post-Bayesian. 
TABLE I

Statistics of the DifFerences Between DaILy SSS MaPs From DifFERENT SMOS PRODUCTS AND ARGO

\begin{tabular}{|c|c|c|c|c|c|c|c|c|c|}
\hline \multirow{3}{*}{ Region } & \multicolumn{8}{|c|}{ Ascending satellite overpasses } \\
\cline { 2 - 10 } & \multicolumn{2}{|c|}{ unfiltered v662 } & \multicolumn{3}{c|}{ v662 } & \multicolumn{3}{c|}{ Post Bayesian } \\
\cline { 2 - 10 } & mean & std & RMS & mean & std & RMS & mean & std & RMS \\
\hline $60^{\circ} \mathrm{S}-60^{\circ} \mathrm{N}$ & -0.08 & 1.18 & 1.20 & 0.01 & 0.76 & 0.77 & 0.05 & 1.11 & 1.11 \\
$60^{\circ} \mathrm{S}-40^{\circ} \mathrm{S}$ & -0.28 & 1.74 & 1.80 & -0.18 & 1.15 & 1.23 & -0.15 & 1.34 & 1.40 \\
$40^{\circ} \mathrm{N}-60^{\circ} \mathrm{N}$ & -0.37 & 2.43 & 2.61 & 0.21 & 1.19 & 1.43 & -0.05 & 1.45 & 1.59 \\
\hline & \multicolumn{8}{|c|}{ Descending satellite overpasses } \\
\cline { 2 - 10 } & \multicolumn{8}{|c|}{ unfiltered v662 } & \multicolumn{7}{c|}{ v662 } & \multicolumn{3}{c|}{ Post Bayesian } \\
\cline { 2 - 10 } Region & mean & std & RMS & mean & std & RMS & mean & std & RMS \\
\hline $60^{\circ} \mathrm{S}-60^{\circ} \mathrm{N}$ & -0.10 & 1.21 & 1.23 & 0.02 & 0.78 & 0.79 & 0.06 & 1.11 & 1.12 \\
$60^{\circ} \mathrm{S}-40^{\circ} \mathrm{S}$ & -0.17 & 1.62 & 1.68 & -0.06 & 1.13 & 1.21 & -0.19 & 1.33 & 1.41 \\
$40^{\circ} \mathrm{N}-60^{\circ} \mathrm{N}$ & -0.90 & 2.57 & 2.85 & -0.20 & 1.35 & 1.62 & -0.23 & 1.45 & 1.62 \\
\hline
\end{tabular}

Table I describes the statistics for ascending (top) and descending (bottom) satellite overpasses corresponding to the unfiltered SSS v662 (first column); the SSS v662 after filtering (second column); and the post-Bayesian product (third column). For each product, the mean, standard deviation (std), and the root mean square (rms) are provided. For the computation of those statistics, we use all SMOS-Argo collocations that are available for each dataset. Therefore, the set of collocations are different for each SMOS product. In the case of ascending overpasses, the mean differences of SMOS and Argo in the global region are very close to zero in the three products: -0.08 for SSS v662 unfiltered; 0.01 for SSS v662; and 0.05 for the post-Bayesian product. In the Northern Ocean, the official L2 product still has residual biases. The SSS v662 unfiltered has a mean bias of -0.37 , which is reduced to 0.21 after filtering out invalid retrievals. In the Southern ocean, the L2 official SSS also has a residual bias, but in this case it is lower than in the Northern Ocean: -0.28 for SSS v662 unfiltered; and -0.18 after applying the filtering criteria. In this case, the post-Bayesian analysis also shows residual bias, which is slightly lower than that of the SSS v662 ( -0.15 versus -0.18$)$. In the case of descending overpasses, differences in the mean bias in the global ocean are present between the three SMOS products. In this case, the unfiltered SSS v662 presents a bias of -0.1 , which after filtering is reduced to 0.02 . The post-Bayesian mean bias is 0.06 (similar to that of ascending overpasses in this region). In the Northern Ocean SSS v662 unfiltered product presents a large bias $(-0.9)$, which is partially compensated after filtering $(-0.20)$. The postBayesian product presents a similar (slightly larger) bias than the filtered SSS v662 ( -0.23 versus -0.20$)$. In the Southern Ocean the biases of the unfiltered SSS v662 and the post-Bayesian are very similar $(-0.17$ and -0.19 , respectively). The filtered SSS v622 reduces the bias up to -0.06 . In all three regions the lowest standard deviation of the difference between SMOS and Argo are given by the filtered SSS v662, and the largest one is provided by the unfiltered one.

Fig. 10 shows the time evolution of the rms SSS for the three SMOS L2 products (ascending overpasses on the left and descending on the right) in the three regions of study: Global (top); NOC (middle); and SOC (bottom). The rms corresponding to unfiltered SSS v662 is always the largest (blue line) and the rms corresponding to the filtered SSS v662 (purple line) is typically the lowest. In the global ocean, the rms of the post-Bayesian product (green line) is slightly lower than the rms of the unfiltered SSS v662 (blue line) and clearly larger than the filtered SSS v662. However, in the Northern and Sourthern oceans, the green line (post-Bayesian) becomes closer to the purple one (filtered SSS v662), which means that in those regions the post-Bayesian analysis reaches a more similar quality to that of the one of the filtered SSS v662 while improving SSS spatial coverage.

Comparisons among the previous results are conditional, to the extent that the coverage of the three products is different, and therefore, the matching pairs of SMOS SSS and Argo data that are considered in the statistics of each dataset are also different. Table II also describes the statistics of the differences between SMOS and Argo, but in this case we compute the statistics over the same set of collocations. Therefore, we consider the following two post-Bayesian comparisons.

1) Post-Bayesian (1): corresponds to the SSS post-Bayesian values in those grid points where both post-Bayesian and unfiltered SSS v662 are available.

2) Post-Bayesian (2): corresponds to the SSS post-Bayesian values in those grid points where both post-Bayesian and filtered SSS v662 are available.

Let us analyze first the results for ascending overpasses. In the global ocean, the comparison between unfiltered SSS v662 and post-Bayesian SSS over the same geo-locations provides very similar performance in terms of biases, but the post-Bayesian SSS analysis produces slightly larger standard deviations than the unfiltered SSS v662 product. In the Sourthern Ocean, the post-Bayesian analysis yields better metrics than the unfiltered SSS v662 one in terms of bias $(-0.15$ versus -0.32$)$, but similar standard deviations (1.34 versus 1.37$)$. While in the Northern Ocean, biases are similar in the two products $(0.05$ for unfiltered SSS v662 and -0.05 for the post-Bayesian), the standard deviation of the post-Bayesian is lower than that of the unfiltered SSS v662 (1.42 and 1.61, respectively). In the comparison between post-Bayesian and filtered SSS v622, the latter always provides the lowest standard deviation. The post-Bayesian product also has larger bias in the global ocean than that of the filtered SSS v662. However, in the Northern and Southern oceans, the post-Bayesian produces the lowest biases. Regarding descending overpasses, in the comparison between unfiltered SSS v662 and post-Bayesian SSS, the performances are similar in the global and Southern oceans, but in the Northern ocean a noticeable improvement is observed with the post-Bayesian product. 


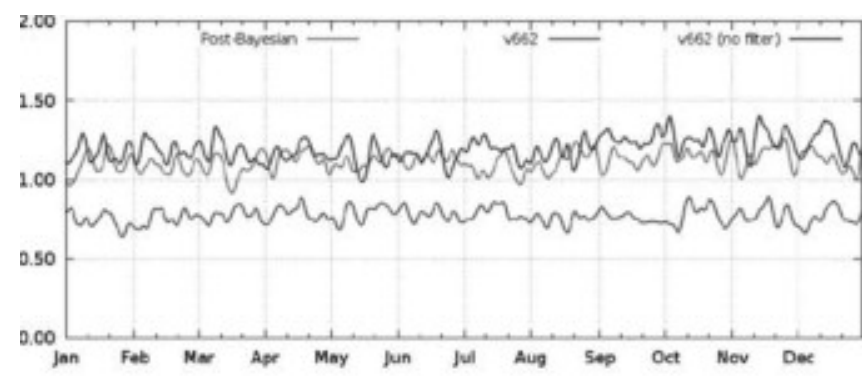

(a)

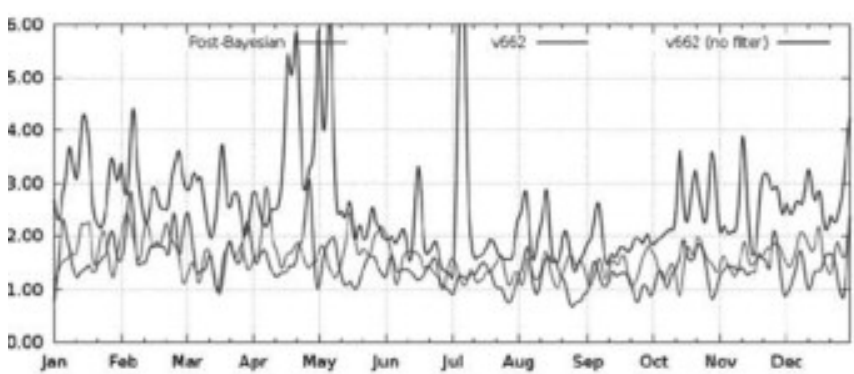

(c)

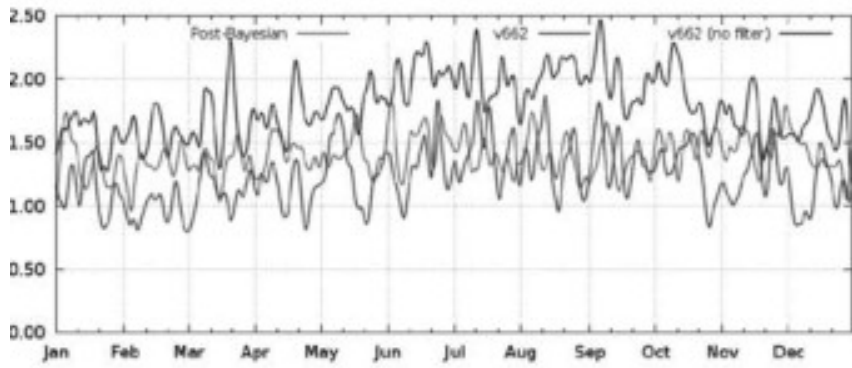

(e)

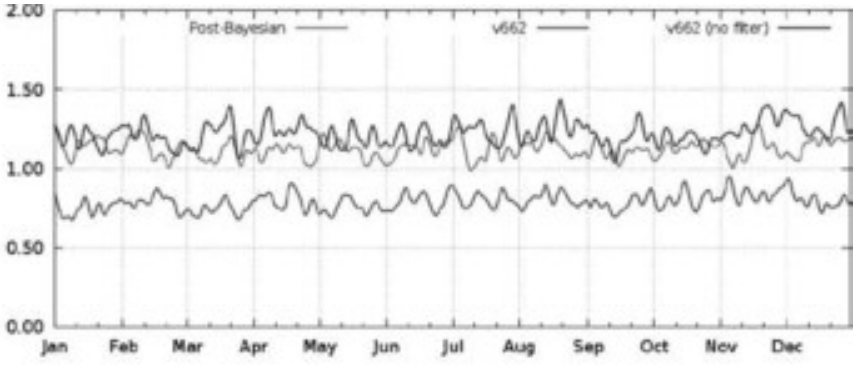

(b)

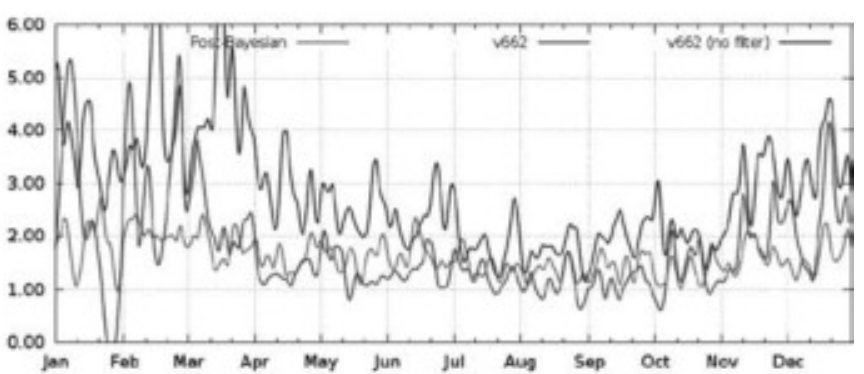

(d)

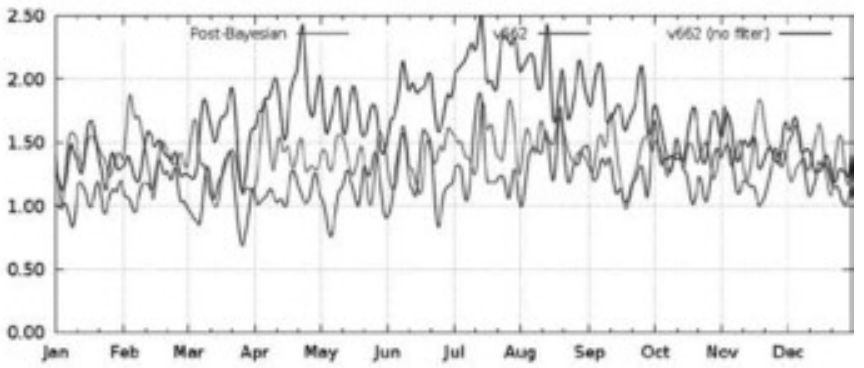

(f)

Fig. 10. Time evolution of the rms of the difference between SMOS SSS and Argo salinity ([psu]), for the three SMOS SSS products. (a) Global (ASC). (b) Global (DES). (c) Northern Ocean (ASC). (d) Northern Ocean (DES). (e) Southern Ocean (ASC). (f) Southern OCean (DES).

TABLE II

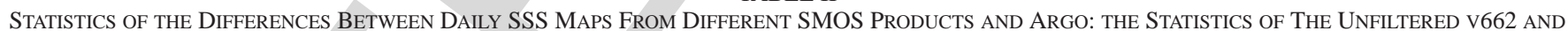
Post-Bayesian Products (1) Are Obtained Over the SAme Set of SMOS and Argo Collocations; the Statistics of the v662 and Post-Bayesian Products (2) ARE ObTained Over the SAME SET OF SMOS AND ARgo COllocations

Ascending satellite overpasses

\begin{tabular}{|c|c|c|c|c|c|c|c|c|c|c|c|c|}
\hline \multirow[b]{3}{*}{ Region } & \multicolumn{12}{|c|}{ Ascending satellite overpasses } \\
\hline & \multicolumn{3}{|c|}{ unfiltered v662 } & \multicolumn{3}{|c|}{ Post Bayesian (1) } & \multicolumn{3}{|c|}{ v662 } & \multicolumn{3}{|c|}{ Post Bayesian (2) } \\
\hline & mean & std & RMS & mean & std & RMS & mean & std & RMS & mean & std & RMS \\
\hline $60^{\circ} \mathrm{S}-60^{\circ} \mathrm{N}$ & -0.06 & 0.98 & 0.99 & 0.07 & 1.08 & 1.09 & 0.01 & 0.73 & 0.74 & 0.11 & 0.98 & 0.99 \\
\hline $60^{\circ} \mathrm{S}-40^{\circ} \mathrm{S}$ & -0.32 & 1.37 & 1.46 & -0.15 & 1.34 & 1.40 & -0.19 & 1.06 & 1.16 & -0.12 & 1.24 & 1.34 \\
\hline $40^{\circ} \mathrm{N}-60^{\circ} \mathrm{N}$ & 0.05 & 1.61 & 1.78 & -0.05 & 1.42 & 1.57 & 0.29 & 1.09 & 1.34 & 0.01 & 1.29 & 1.50 \\
\hline \multirow[b]{3}{*}{ Region } & \multicolumn{12}{|c|}{ escending satellite overpasses } \\
\hline & \multicolumn{3}{|c|}{ unfiltered v662 } & \multicolumn{3}{|c|}{ Post Bayesian (1) } & \multicolumn{3}{|c|}{ v662 } & \multicolumn{3}{|c|}{ Post Bayesian (2) } \\
\hline & mean & std & RMS & mean & std & RMS & mean & std & RMS & mean & std & RMS \\
\hline $60^{\circ} \mathrm{S}-60^{\circ} \mathrm{N}$ & -0.05 & 1.02 & 1.03 & 0.06 & 1.10 & 1.11 & 0.01 & 0.75 & 0.76 & 0.12 & 0.99 & 1.01 \\
\hline $60^{\circ} \mathrm{S}-40^{\circ} \mathrm{S}$ & -0.20 & 1.32 & 1.40 & -0.19 & 1.33 & 1.40 & -0.08 & 1.04 & 1.13 & -0.18 & 1.26 & 1.37 \\
\hline $40^{\circ} \mathrm{N}-60^{\circ} \mathrm{N}$ & -0.33 & 1.83 & 2.04 & -0.21 & 1.44 & 1.60 & -0.13 & 1.18 & 1.47 & -0.19 & 1.26 & 1.52 \\
\hline
\end{tabular}

On the contrary, in the comparison of filtered SSS v662 and post-Bayesian SSS, the first provides the best performance.

In order to analyze how the residual errors that are still present in the L2 SSS products decrease when they are averaged in L3 maps, we also compute monthly SSS maps and we compare them with Argo. The statistics of the differences with respect to Argo are described in Table III. The comparisons are performed over each corresponding dataset of collocations (so different coverages are considered). The maps computed from unfiltered SSS v662 provide the worst metrics in terms of bias, std, and rms. 
TABLE III

STATISTICS OF THE DifFERENCES BETWEEN MONTHLY SSS MAPS From DifFERENT SMOS PRODUCTS SAND ARGO

\begin{tabular}{|c|c|c|c|c|c|c|c|c|c|}
\hline & \multicolumn{8}{|c|}{ Ascending satellite overpasses } \\
\cline { 2 - 10 } Region & \multicolumn{3}{|c|}{ unfiltered v662 } & \multicolumn{3}{c|}{ v662 } & \multicolumn{3}{c|}{ Post Bayesian } \\
\cline { 2 - 10 } & mean & std & RMS & mean & std & RMS & mean & std & RMS \\
\hline $60^{\circ} \mathrm{S}-60^{\circ} \mathrm{N}$ & -0.12 & 0.52 & 0.54 & -0.02 & 0.40 & 0.41 & 0.03 & 0.46 & 0.46 \\
$60^{\circ} \mathrm{S}-40^{\circ} \mathrm{S}$ & -0.38 & 0.55 & 0.68 & -0.22 & 0.54 & 0.60 & -0.18 & 0.49 & 0.53 \\
$40^{\circ} \mathrm{N}-60^{\circ} \mathrm{N}$ & -0.12 & 0.92 & 0.98 & 0.25 & 0.71 & 0.79 & -0.03 & 0.63 & 0.65 \\
\hline & \multicolumn{8}{|c|}{ Descending satellite overpasses } \\
\cline { 2 - 10 } & \multicolumn{8}{|c|}{ unfiltered v662 } & \multicolumn{7}{c|}{ v662 } & \multicolumn{3}{c|}{ Post Bayesian } \\
\cline { 2 - 10 } Region & mean & std & RMS & mean & std & RMS & mean & std & RMS \\
\hline $60^{\circ} \mathrm{S}-60^{\circ} \mathrm{N}$ & -0.13 & 0.54 & 0.56 & -0.01 & 0.43 & 0.43 & 0.04 & 0.48 & 0.48 \\
$60^{\circ} \mathrm{S}-40^{\circ} \mathrm{S}$ & -0.83 & 1.20 & 1.49 & -0.19 & 0.87 & 0.98 & -0.17 & 0.63 & 0.70 \\
$40^{\circ} \mathrm{N}-60^{\circ} \mathrm{N}$ & -0.24 & 0.54 & 0.64 & -0.06 & 0.53 & 0.56 & -0.20 & 0.37 & 0.42 \\
\hline
\end{tabular}

In the global ocean, the post-Bayesian and the filtered v662 maps provide similar performance, but the post-Bayesian produced a slightly larger std and rms (0.41 versus 0.46$)$. However, all the metrics are better for the post-Bayesian analysis in the Northern and Southern oceans. Only the bias of the post-Bayesian map for descending overpasses is larger than that of the filtered v622 L3 map in the Northern ocean, but the global rms is still lower in the post-Bayesian case.

In Fig. 11, differences between one month (August) of SMOS and the annual WOA2013 are shown for the three SMOS products, and for ascending (left) and descending (right) overpasses. The monthly maps reflect the results discussed on a daily time scale: the unfiltered v662 seem to be affected by residual biases that are partially compensated after filtering. However, the coverage of the filtered v662 product does not allow valid SSS to be retrieved in the European and Asian coasts, where the postBayesian analysis is providing valid SSS. Indeed, the regions poorly covered or that have spurious anomaly values seem to be quite coincident with the regions where distributions are more degraded in terms of symmetry and flatness (see Fig. 8).

\section{DISCUSSION AND CONCLUSION}

In this paper, we empirically characterize SMOS TB biases and uncertainties over the ocean, profiting from a consistent SMOS time series. Regarding the biases, this study is focused on those biases that are constant in time. We show that SMOS TB biases are not only dependent on the position in the field of view and the orbit direction of the acquisition (which is the paradigm of the standard OTT correction used in the official L2OS processor), but also strongly depend on the geographical location of the acquisition. Empirical geographically dependent OTTs are estimated and differences between these new OTT and the standard OTT are analyzed. We demonstrate that the standard OTT correction has severe limitations in coastal regions and at high latitudes. This is a known finding. An LSC correction is currently being applied in the official SMOS L2OS processor (v662). This has led to a significant improvement in the SMOS L2OS SSS salinity with respect to the previous version (v622). However, there are still some issues in the current SMOS L2OS SSS v662 products affecting SSS retrievals mainly in semi-enclosed seas and high latitudes. These regions coincide with regions that we show are poorly corrected by the standard OTT (see Fig. 2).
The uncertainty of the SMOS TB over the ocean is also empirically characterized. Once the acquisition conditions are fixed (i.e., the orbit direction, the position in the field of view, and the geographical location), the empirical SMOS TB uncertainty is separated into three contributions: the theoretical radiometric accuracy, the geophysical variability, and an excess of uncertainty. We show that this excess of uncertainty is also geographically dependent. Regions strongly affected by RFI typically present a large excess of uncertainty. Besides, coastal areas are also affected by an increase of excess of uncertainty, new finding. This excess of uncertainty is an additional random error not characterized by the expected radiometric accuracy and by the observed geophysical variability. In the Bayesian approach implemented in the official L2 processor, the cost function is weighted by the theoretically computed radiometric accuracy and a constant value which is equal for all geographical locations. The use of the estimated excess of uncertainty (not constant at all locations, see Fig. 4) in the cost function could lead to better salinity retrievals, particularly in those regions where the excess of uncertainty is larger (namely in coastal and RFI-affected regions).

In the study of the SMOS errors over the ocean, the third- and fourth-order moments of the salinity statistical distributions that are retrieved under the same acquisition conditions were analyzed. The skewness and the kurtosis provide information about the asymmetry and the peakedness of the distributions. Both metrics together give an indicator of how far these distributions vary in their accuracy. We show that at high latitudes (northern and southern) regions and in the European and Asian coasts, as well as in regions close to Madagascar, Chinese Sea, and part of the North Atlantic, distributions are typically degraded. Those regions are typically affected by errors that are not constant in time. At high latitudes, the effect of the eclipse lasts typically only a few months. During this period, strong negative biases degrade the quality of the retrievals at high latitudes (see [7, Fig. 6]). On time, they could affect the peakedness or/and the skewness of the distributions at those locations. Non-permanent could similarly affect the accuracy of those distributions because, as in the previous case, they degrade the retrievals over a short period (not constant) of time. This would translate into temporal changes in both the magnitude and bias, and could explain why such high-latitude regions show a non-accurate distribution. RFI sources may change their intensity, which could translate into temporal changes in the magnitude of the biases not only 

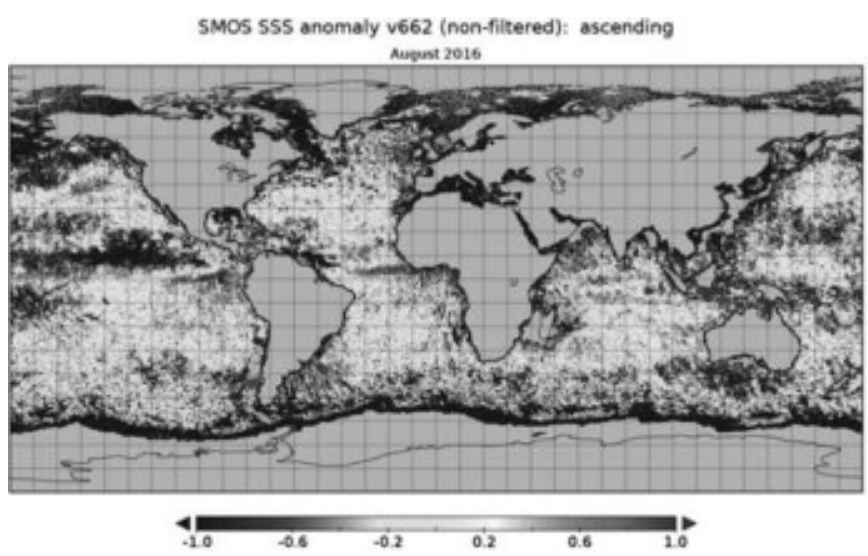

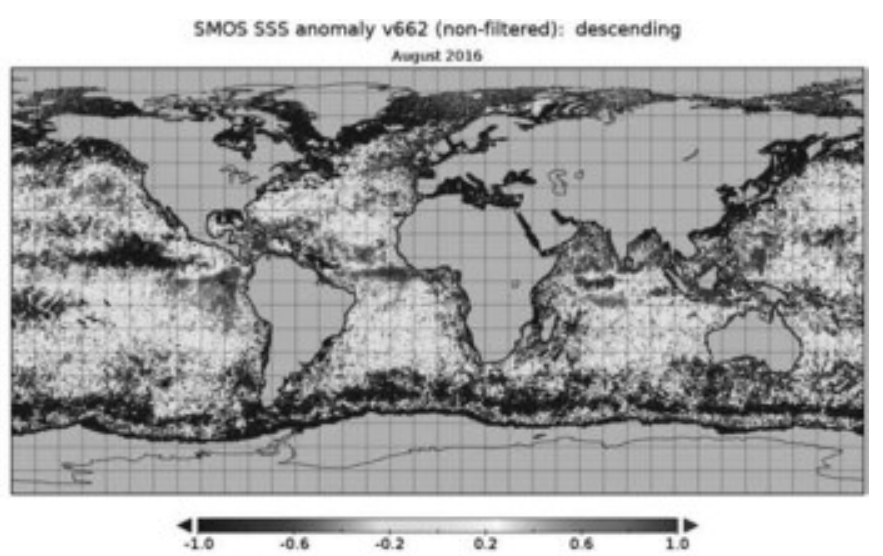

(a)

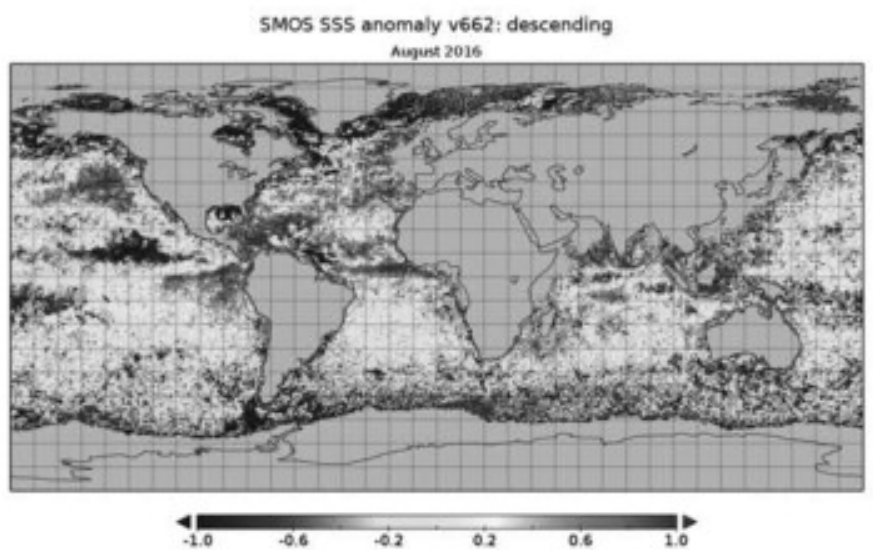

(b)

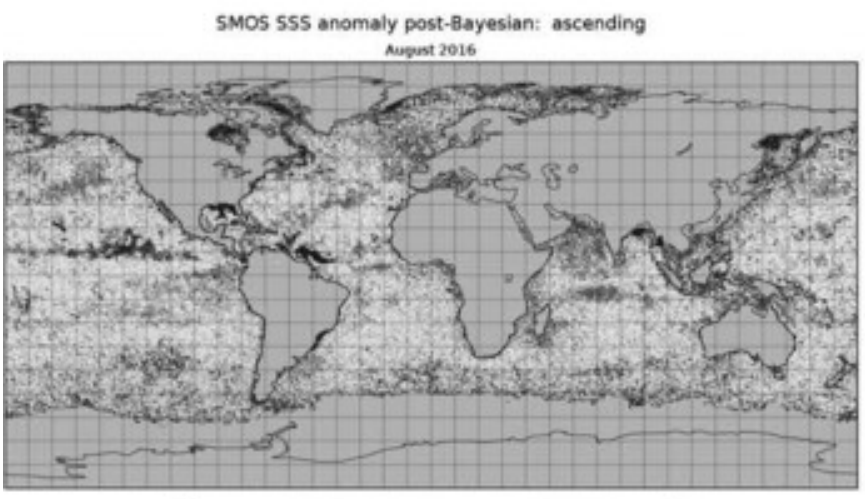

\begin{tabular}{lllllll}
\hline & 0.6 & -0.2 & 0.2 & 0.6 & 10
\end{tabular}
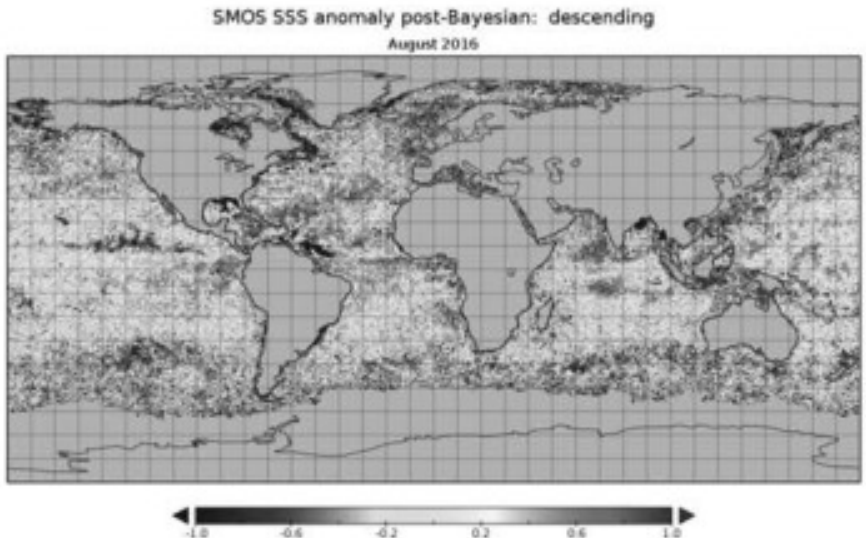

(c)

Fig. 11. Monthly maps of the SMOS SSS anomaly. (a) Unfiltered v662. (b) v662. (c) Post-Bayesian.

non-regular on time biases but also non-regular in magnitude. This could explain why such regions show more degraded distributions.

Based on these results, a new Level 2 SMOS SSS product is proposed: the post-Bayesian L2 SSS. This product is not corrected by the standard OTT; instead we use the empirical characterization of the biases presented in Section II. The corrected salinity retrievals are then averaged by using the 789 empirical characterization of the uncertainty presented in 790 Section III. The analysis of the statistical third- and fourth-order 791 moments is also used in order to define filtering criteria that are 792 used to discard degraded salinity values. Although this is sub- 793 ject to further improvements, it is currently a viable product. We 794 compare the post-Bayesian SSS product with the last version of 795 
the official Level 2 SSS product (v662). The latter, when no additional filtering is applied, is worse in terms of bias and standard deviation than the post-Bayesian product with respect to Argo in situ salinities. The improvement produced by the post-Bayesian analysis is noticeable at high latitudes. We show that in regions such as the Mediterranean Sea and the Chinese seas, the Level 2 SSS v662 product provides unreliable salinity values, while the post-Bayesian one allows the retrieval of geophysically reasonable salinity values. The Level 2 SSS UDP v662 provides quality flags that allow values of the worst quality to be discarded. By applying the quality flags described in [22], we generate the filtered L2 v662 product. When we compare the filtered L2 v662 product with the post-Bayesian one, we observe that the level of noise of the filtered L2 v662 product is noticeable lower than that of the post-Bayesian product, but the coverage of the former is dramatically reduced in general; especially at high latitudes and in semi-enclosed seas, but also close to the coastal regions. However, we consider this tradeoff to be acceptable.

The comparison with Argo in these regions at Level 2 is difficult because the number of available Argo data on daily time scales is typically low and this may produce non-significant statistics. Recent works show that at Level 3, the non-Bayesian (or post-Bayesian) scheme provides salinity retrievals with reasonable accuracy in the Mediterranean Sea [24] and in the Arctic Ocean [25]. Therefore, we have extended the validation of these products to monthly L3 maps. We show in this case that the level of noise of the post-Bayesian and that of the filtered L2 v662 is similar in medium and low latitudes, but the post-Bayesian noise level is lower at high latitudes. Non-filtered products still contain some kind of systematic error that cannot be reduced by averaging. Furthermore, the coverage of L3 monthly maps computed from the filtered L2 v662 (see Fig. 11) well coincides with those regions where we show that the distributions are more accurate (i.e., more symmetric and peaked) (see Fig. 8). This suggests that the information about the third- and fourthorder moments of those distributions can be used for defining a more appropriated filtering criteria in the official L2 processor; to better discard all degraded TBs that lead to non-valid salinity retrievals.

Future work to improve this L2 post-Bayesian product (and therefore, also the quality of the L3 SSS product) is focused on the application of the so-called nodal sampling ( see [26] and [27]), which has been shown to improve the quality of salinity retrievals in strongly RFI-contaminated regions [28].

\section{ACKNOWLEDGMENT}

The authors would like to thank Dr. D. Burrage for the extensive review of this paper. His suggestions have significantly improved the quality of the paper.

\section{REFERENCES}

[1] J. Font, A. Camps, and J. Ballabrera-Poy, "Microwave Aperture Synthesis Radiometry: Setting the Path for Sea Surface Salinity Measurements from Space," in Remote Sensing of European Seas. New York, NY, USA: Springer-Verlag, 2008.
[2] S. Mecklenburg, N. Wright, C. Bouzina, and S. Delwart, "Getting down to business-SMOS operations and products," ESA Bull., vol. 137, pp. 25-30, 2009.

[3] Y. Kerr et al., "The SMOS mission: New tool for monitoring key elements of the global water cycle," Proc. IEEE, vol. 98, no. 5, pp. 666-687, May 2010.

[4] K. McMullan et al., "SMOS: The payload," IEEE Trans. Geosci. Remote Sens., vol. 46, no. 3, pp. 594-605, Mar. 2008.

[5] S. Zine et al., "Overview of the SMOS sea surface salinity prototype processor," IEEE Trans. Geosci. Remote Sens., vol. 46, no. 3, pp. 621-645, Mar. 2008.

[6] C. Gabarro, M. Portabella, M. Talone, and J. Font, "Toward an optimal SMOS ocean salinity inversion algorithm," IEEE Geosci. Remote Sens. Lett., vol. 6, no. 3, pp. 509-513, Jul. 2009.

[7] M. Martín-Neira et al., "SMOS Instrument performance and calibration after six years in orbit," Remote Sens. Environ., vol. 180, pp. 19-39, 2016.

[8] J. Tenerelli and N. Reul, "Analysis of L1PP calibration approach impacts in SMOS Tbs and 3-Days SSS retrievals over the pacific using an alternative ocean target transformation applied to L1OP data," IFREMER/CLS, Tech. Rep., Feb. 2010.

[9] E. Olmedo et al., "Improving time and space resolution of SMOS salinity maps using multifractal fusion," Remote Sens. Environ., vol. 180, pp. 246-263, 2016.

[10] N. Kolodziejczyk, J. Boutin, J. Vergely, S. Marchand, N. Martin, and G. Reverdin, "Mitigation of systematic errors in SMOS sea surface salinity," Remote Sens. Environ., vol. 180, pp. 164-177, 2016.

[11] J. Boutin et al., "New SMOS Sea Surface Salinity with reduced systematic errors and improved variability," Remote Sens. Environ., vol. 214, pp. 115-134, 2018 .

[12] E. Olmedo, J. Martinez, A. Turiel, J. Ballabrera-Poy, and M. Portabella, "Debiased non-Bayesian retrieval: A novel approach to SMOS sea surface salinity,” Remote Sens. Environ., vol. 193, no. Suppl C, pp. 103-126, 2017.

[13] N. Reul et al., "Analysis of L-band radiometric measurements conducted over the North Sea during the CoSMOS-OS airborne campaign." in Proc. IEEE Int. Geosci. Remote Sens. Symp., 2007.

[14] S. Guimbard, J. Gourrion, M. Portabella, A. Turiel, C. Gabarro, and J. Font, "SMOS semi-empirical ocean forward model adjustment," IEEE Trans. Geosci. Remote Sens., vol. 50, no. 5, pp. 1676-1687, May 2012.

[15] J. Sabater and P. De Rosnay, "Tech note - parts 1/2/3: Operational preprocessing chain, collocation software development and offline monitoring suite," , ECMWF, Tech. Rep., 2010.

[16] J. Tenerelli, N. Reul, A. Mouche, and B. Chapron, "Earth-viewing L-band radiometer sensing of sea surface scattered celestial sky radiation-Part I: General characteristics," IEEE Trans. Geosci. Remote Sens., vol. 46, no. 3, pp. 659-674, Mar. 2008. [Online]. Available: http://dx.doi.org/10.1109/TGRS.2007.914803

[17] National Oceanographic Data Center, WOA 2013 V2 Data Access. 2013 [Online]. Available: https://www.nodc.noaa.gov/cgi-bin/OC5/woa13/ woa13.pl, Accessed on: Aug. 23, 2016.

[18] M. M. Zweng et al., World Ocean Atlas 2013, Volume 2: Salinity (NOAA Atlas NESDIS 74), Levitus, Ed., A. Mishonov Technical Ed. Silver Spring, MD, USA: NOAA, 2013.

[19] C. Swift and R. McIntosh, "Considerations for microwave remote sensing of ocean-surface salinity," IEEE Trans. Geosci. Electron., vol. GE-21, no. 4, pp. 480-491, Oct. 1983.

[20] S. Yueh, R. West, W. Wilson, F. Li, S. Nghiem, and Y. Rahmat-Samii, "Error sources and feasibility for microwave remote sensing of ocean surface salinity," IEEE Trans. Geosci. Remote Sens., vol. 39, no. 5, pp. 1049-1059, May 2001.

[21] M. Talone, M. Portabella, J. Martnez, and V. Gonzlez-Gambau, "About the optimal grid for SMOS level 1c and level 2 products," IEEE Geosci. Remote Sens. Lett., vol. 12, no. 8, pp. 1630-1634, Aug. 2015.

[22] BEC Team, SMOS-BEC Ocean and Land Products Description BECSMOS-0001-PD, Barcelona Expert Centre. Jun. 2015. [Online]. Available: http://cp34-bec.cmima.csic.es/doc/BEC-SMOS-0001-PD.pdf

[23] J. Boutin, N. Martin, X. Yin, J. Font, N. Reul, and P. Spurgeon, "First assessment of SMOS data over open ocean: Part ii sea surface salinity," IEEE Trans. Geosci. Remote Sens., vol. 50, no. 5, pp. 1662-1675, May 2012, doi: 10.1109/TGRS.2012.2184546.

[24] E. Olmedo, I. Taupier-Letage, A. Turiel, and A. Alvera-Azcárate, "Improving SMOS sea surface salinity in the Western Mediterranean sea through multivariate and multifractal analysis," Remote Sens., vol. 10, no. 3, p. $485,2018$.

[25] E. Olmedo et al., "Seven years of SMOS sea surface salinity at high latitudes: Variability in Arctic and sub-Arctic regions," Remote Sens. 


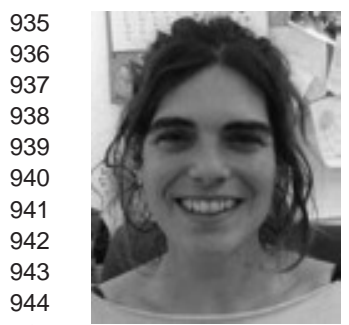

Estrella Olmedo was born in La Línea de la Concepción, Spain, in 1979. She received the B.Sc. and Ph.D. degrees in mathematics from the University of Barcelona, Barcelona, Spain, in 2001 and 2007, respectively.

Her research started in the field of dynamical systems and applied mathematics in the context of computation of quasi-invariant tori. Then, she was with Deimos Space S.L.U in European Space Agency's (ESA) projects related with the design of the future European Space Surveillance System. Since 2012, she has been a Researcher with the Physical Oceanography Department, Institut de Ciencies del Mar, Consejo Superior de Investigaciones Cientificas, Barcelona, Spain. Her current research includes signal and data processing of the Soil Moisture and Ocean Salinity (SMOS) data, studies of oceanographic applications of the remotely sensed salinity, and analysis of the marine turbulence.

Dr. Olmedo is also a Member of the Barcelona Expert Center, Barcelona.
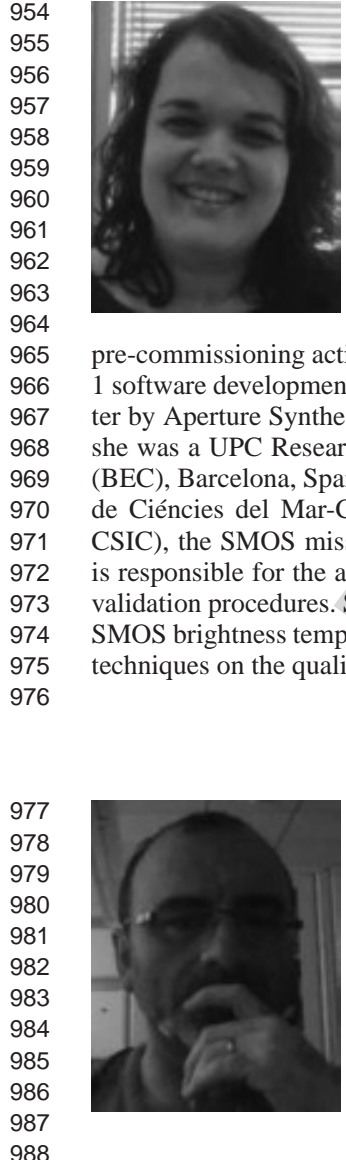

Verónica González-Gambau was born in Huesca, Spain, in 1981. She received the M.S. and Ph.D. degrees in telecommunication engineering from the School of Telecommunication Engineering, Universitat Politcnica de Catalunya (UPC), Barcelona, Spain, in 2006 and 2012, respectively.

In 2006, she joined the Passive Remote Sensing Group of the Signal Theory and Communications Department, UPC, where she was involved in the Soil Moisture and Ocean Salinity (SMOS) payload onground characterization in the framework of SMOS pre-commissioning activities. She was also collaborating with the SMOS Level 1 software development and data analysis of the Microwave Imaging Radiometer by Aperture Synthesis (MIRAS) instrument validation campaigns. In 2007, she was a UPC Researcher in collaboration with the Barcelona Expert Center (BEC), Barcelona, Spain. She is currently a Research Scientist with the Institut de Ciéncies del Mar-Consejo Superior de Investigaciones Científicas (ICMCSIC), the SMOS mission coleading institution, Barcelona, Spain, where she is responsible for the advance Level 1 error correction techniques and in-orbit validation procedures. She is working on the development of algorithms for the SMOS brightness temperatures improvement, and assessing the impact of these techniques on the quality of the geophysical retrievals.

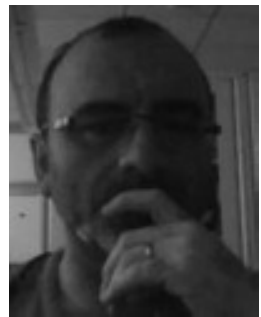

Antonio Turiel was born in Len, Spain, in 1970. He received the B.Sc. degree in physics, the B.Sc. degree in mathematics, and the Ph.D. degree in theoretical physics from the Autonomous University of Madrid, Madrid, Spain, in 1993, 1994, and 1998, respectively.

$\mathrm{He}$ is currently with the Department of Physical Oceanography, Institut de Cincies del Mar, Barcelona, Spain. His research interests include signal and image processing applied to remote sensing of the oceans, marine turbulence at mesoscale, and ocean circulation at different scales.

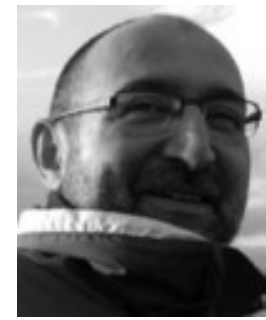

Justino Martínez was born in Girona, Spain, in 1966. He received the B.Sc. and Ph.D. degrees in physics from the Autonomous University of Barcelona, Barcelona, Spain, in 1991 and 1995, respectively.

His research started in the field of relativistic thermodynamics in the context of gravitational collapse. He is currently a Scientific Programmer with the Physical Oceanography Department, Institut de Ciencies del Mar, Consejo Superior de Investigaciones Cientificas, Barcelona, Spain. His current work is centered in SMOS L1 corrections, in the research concerning sea-ice products development and in the improvement of the Arctic sea surface salinity retrieval.

Dr. Martínez is also a Member of the Barcelona Expert Center, Barcelona and he has been involved in the L3 and L4 SMOS products generation and validation and forward model implementation.

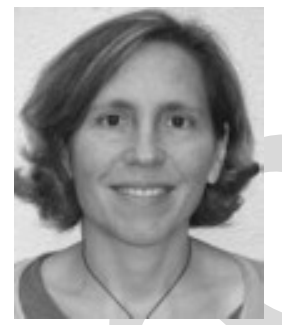

Carolina Gabarró was born in Barcelona, Spain, in 1974. She received the B.Eng. degree in telecommunications engineering and the Ph.D. degree in ocean science from the Universitat Politcnica de Catalunya Barcelona, Spain, in 1998 and 2004, respectively.

From 1997 to 1999, she was with ESTEC, ESA Since 2000, she has been with the Physical Oceanography Department, Instituto de Ciencias del Mar, CSIC, Barcelona, Spain. Her research interests include brightness temperature modeling and inversion algorithms for remote sensing passive microwave radiometer for Cryospheric applicactions.

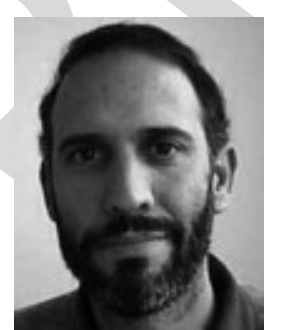

Marcos Portabella was born in Barcelona, Spain, in 1970. He received the B.Sc. degree in physics from the University of Barcelona, Barcelona, Spain, in 1994, the M.Sc. degree in remote sensing from the Institute of Space Studies of Catalonia, Barcelona, Spain, in 1995, and the Ph.D. degree in physics from the University of Barcelona in 2002.

He is currently with the Institut de Cincies del Mar, Barcelona, Spain, focusing on satellite remote sensing. In particular, he is involved in scatterometry and L-band radiometry.

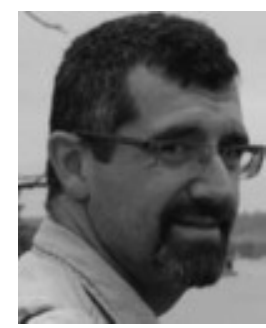

Joaquim Ballabrera-Poy received the B.Sc. degree in physics, and the Ph.D. degree in geophysics. Head of the Department of Physical and Technological Oceanography, Barcelona, Spain. He is an Exper in data assimilation, numerical modeling, data analysis, and operational oceanography. He is an author of more than 50 articles published in SCI journals and participated in 22 research projects, acting as a PI in 5 of them. He was a WP leader in FP7 projects: MyOCEAN and E-AIMS

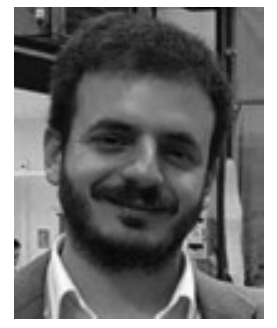

Manuel Arias was born in Madrid, Spain, in 1981. He received the B.Sc. degree in marine sciences (esp. physical oceanography) and the M.Sc. degree in remote sensing and physical oceanography, both from the University of Cadiz, Cadiz, Spain, in 2004 and 2008, respectively.

He worked in research projects within the Remote Sensing Group of the Applied Physics Department till 2013. He has been with ARGANS, Plymouth, U.K., since 2013 and is currently a Senior Project Manager and Senior Earth Observation Scientist there. His research interests include development of retrieval algorithms for remote sensing data, remote sensing applied to physical oceanography, with special focus in teleconnections, climate, and extremes.
1043 1044 


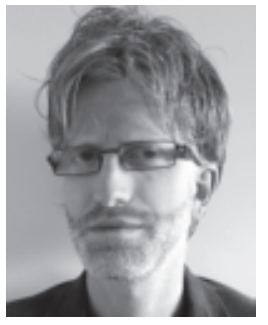

Roberto Sabia (M'03) was born in Napoli, Italy, in 1975. He received the graduate degree (cum laude) in environmental sciences, curriculum in oceanography, from Universit Parthenope in Napoli, Naples, Italy, in 2002 , and the Ph.D. (cum laude) degree in signal theory and communication from Universitat Politecnica de Catalunya, Barcelona, Spain, in 2008, with a thesis on the ocean salinity retrieval within the ESA Soil Moisture and Ocean Salinity (SMOS) mission.

In summer 2006, he was a Visiting Ph.D. Student with the National Oceanography Centre, Southampton, U.K. From 2008 to 2010, he was a postdoc with the Barcelona Expert Centre (SMOS-BEC), Institut de Cincies del Mar (ICM-CSIC), Barcelona, Spain. From 2010 to 2013, he was an ESA postdoc Research Fellow with ESA-ESRIN, Frascati, Italy. In 2013, he joined Telespazio-Vega UK Ltd. being based at ESAESTEC, Noordwijk, the Netherlands. Since 2015, he has been with ESA-ESRIN, Frascati, Italy, with management and coordination responsibilities on several SMOS contracts. In 2010, he has led a European COST Action proposal titled SMOS Mission Oceanographic Data Exploitation (SMOS-MODE), successfully funded for the period 2011-2015. His research interests include the ocean remote sensing framework, specifically on ocean salinity, carbon cycle, and ocean acidification.

Dr. Sabia was the recipient of the best Ph.D. awards in remote sensing of the European IEEE GRS Society and Spanish Chapter of the IEEE GRS Society, both in 2008. He received the IEEE GRS Society 2014 Symposium Interactive Prize Paper award. In 2018, he received the APM Project Management Qualification (PMQ).

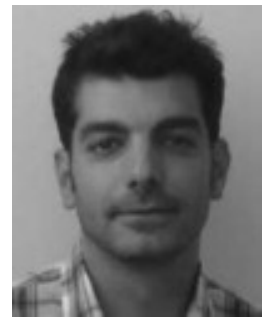

Roger Oliva received the M.Sc. degree in telecommunication engineering from the Polytechnic University of Catalonia, Barcelona, Spain, in 2004.

In his professional live, he has been working in several space and astronomy projects, including the European Space Agency's mission Mars Express, two astronomical microwave observatories, and in the design of advanced telecommunications satellite payloads. Since 2007, he has been with the European Space Agency Earth Observation satellite SMOS, Paris, France. In SMOS, he is the Coordinator of the Calibration and Level 1 processing team and leads the SMOS Radio Frequency Interference team.

Mr. Oliva received the Certificate of Appreciation from the IEEE GRSS FARS Committee for their successful efforts in working with national authorities in removing radio-frequency interference sources from the protected 14000-1427 MHz EESS band in 2013. Since 2017, he is a co-chair of the FARS technical committee.

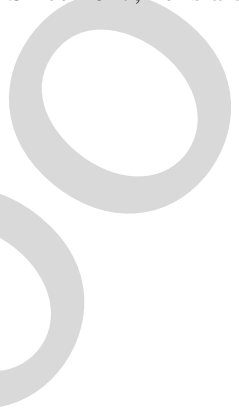

